\title{
Distributed Maximum Maintenance on Hierarchically Divided Graphs
}

\author{
P. J. A. Lentfert and S. D. Swierstra \\ Department of Computer Science, Utrecht University, The Netherlands.
}

Keywords: Distributed algorithms; Hierarchically divided networks; Program development; Stepwise refinement; UNITY

\begin{abstract}
The design and verification of distributed and concurrent algorithms is highly complex, and thus error-prone. It is our experience that the intertwined use of an informal description as well as a formal method in designing and studying an algorithm, is a fruitful one. The advantage of the informal description is the ease with which algorithms can be produced, studied and discussed. In contrast with formal methods however, errors are easily made in informal arguments about programs that seem to be correct, but in fact are not.

In this paper we use an informal argument, carefully augmented with the use of the UNITY formalism, in the design of a distributed algorithm for maintaining the maximum value of a bag of frequently changing integers on a hierarchically divided network. The resulting algorithm is obtained by first designing an abstract algorithm on a virtual datastructure. Next, the abstract algorithm is transformed into the distributed algorithm.
\end{abstract}

\section{Introduction}

Distributed algorithms are often informally described and attempts to derive them in a calculational style are only now slowly taking off. The advantage of a not fully formal description is the seeming ease with which they can be produced and studied.

A major disadvantage in providing only informal arguments is that errors are easily made. It is our experience that distributed algorithms which seemed to be

Correspondence and offprint requests to: Patrick Lentfert, Department of Computer Science, Utrecht University, P.O. Box 80.089, 3508 TB Utrecht, The Netherlands. 
correct, and were even proven to be correct in a not fully formal way, in fact turned to be incorrect.

To ensure correctness, a fully formal proof has to be developed. UNITY [ChM88] is a formalism which is claimed to be helpful in the design of distributed and concurrent programs. In [ChM88] it is claimed that UNITY is a complete theory of programming because it includes the following important aspects:

- It is a method for specification of programs.

- It is a method for reasoning about specifications.

- It is a method of developing programs together with a proof that they meet their specification.

- It is a method of transforming programs to achieve high performance on machines available for their execution.

The formal design of algorithms may be extremely tiresome, although rewarding. Using formal methods (including UNITY) proofs soon become very complex when studying nontrivial problems. Therefore, an informal description may still be helpful.

In this article algorithms are first described informally. A reader can learn about the algorithms by reading the informal parts.

After the informal description of each algorithm, the algorithm is formally described and proven correct using UNITY. The knowledge of the algorithm, which follows from the informal description, makes that the formal derivation and correctness proofs are easier to understand. Moreover, without this knowledge the formal derivations of the algorithms could not have been derived.

Our goal is to formally design a distributed algorithm on a hierarchically divided network, i.e., a network that is divided in domains; each domain, in turn, may be divided in subdomains, etc. The derived algorithm describes how to maintain the maximum value of a bag of frequently changing inputs. Each node in the network is associated with a single element of this bag of inputs, and each node has to maintain the maximum value of the inputs of all nodes.

As a result of hierarchically dividing the network, the storage and update complexity of the algorithm is reduced. It is not necessary to store at each node in the network an item of information for every other node.

In Section 2 a short introduction to UNITY is given. Only so much of the UNITY notation and logic is presented for the reader to be able to understand this paper. In Section 3 the topological model is described which is used in this article. The problem is presented in Section 4. In Section 5 an abstract algorithm operating on a virtual datastructure is developed. This algorithm is subsequently transformed into a distributed algorithm that operates on a hierarchically divided network in Section 6. Because, as stated above, an informal description is helpful, such a description of each algorithm is given, before being formally described and proven correct. In Section 7 concluding remarks are given.

\section{UNITY}

UNITY is a formalism for the development of parallel and distributed programs which was developed by Chandy and Misra [ChM88]. Presently, research in UNITY is still going on [San91]. Program development in UNITY is done by stepwise refinement of specifications, until the specification is restrictive enough 
to be translated into UNITY code. Examples of this approach can be found in [ChM88, Kna90, Mis89, Sta88]. This section presents the most essential aspects of UNITY that are relevant to our discussion. For more information and many examples using it to derive programs, see [ChM88] and [San91].

\subsection{Programs in UNITY}

A UNITY program consists of four parts:

The declare-section is a set of variable declarations.

The initially-section is a set of equations defining initial values for some or all the variables.

The always-section is a set of equations defining variables in terms of other variables.

The assign-section is a set of multiple, conditional assignment statements.

The equations in the initially-section and statements in the assign-section are separated by the operator $\mathrm{C}$. This operator is symmetric, associative and idempotent.

Every statement is of the form

$$
x_{1}, x_{2}, \ldots, x_{n}=e_{1}, e_{2}, \ldots, e_{n} \quad \text { if } B
$$

where each $x_{i}$ is a variable, $e_{i}$ an expression that may depend on all $x_{j}$ 's and $B$ a boolean expression. To define variables by case analysis, as is common practice in mathematics, the symbol $\sim$ is introduced. For example,

$$
x= \begin{cases}e_{1} & \text { if } B_{1} \\ e_{2} & \text { if } B_{2} \\ \vdots & \\ e_{n} & \text { if } B_{n}\end{cases}
$$

is denoted as

$$
\begin{aligned}
& x:=e_{1} \quad \text { if } B_{1} \sim \\
& e_{2} \quad \text { if } B_{2} \sim \\
& e_{n} \quad \text { if } B_{n}
\end{aligned}
$$

If none of the boolean expressions is true, the corresponding variable is left unchanged. If $B_{i}$ and $B_{j}(i \neq j)$ are both true, then $e_{i}=e_{j}$ must hold. This guarantees that every assignment is deterministic.

To denote quantified statements, the following notation is used

$\langle$ पvariable-list : boolean-expression :: statement-list $\rangle$

For example, given arrays $X[0 \ldots n]$ and $Y[0 \ldots n]$ of integers,

$$
\langle\square i: 0 \leq i \leq n:: X[i]:=Y[i]\rangle
$$


is equivalent to

$$
X[0]:=Y[0][X[1]:=Y[1] \square \ldots \square X[n]:=Y[n]
$$

Moreover, to denote quantified expressions, a similar notation is used. For example, given array $X[0 \ldots n]$ of integers,

$$
\langle\max i: 0 \leq i \leq n:: X[i]\rangle
$$

denotes the maximal element in $X[0 \ldots n]$. If there is no instance of the quantification, the value of the quantified expression is the unit element of the operator. For the given example, it would be $-\infty$.

Likewise, $\langle\forall i:: X[i]>0\rangle$ holds if and only if all elements of $X$ are positive. In this case, the range of $i$ is understood from the given context. Moreover, any expression having a free variable, i.e., a variable that is neither bound nor a program variable, is assumed to be universally quantified over all possible values of the variable. E.g., $\langle\forall i \quad: X[i]=k \Rightarrow Y[i] \geq k\rangle$ is a shorthand for $\langle\forall k::\langle\forall i:: X[i]=k \Rightarrow Y[i] \geq k\rangle\rangle$.

The execution of a UNITY program starts in a state in which all variables have the values specified in the initially-section. Uninitialised variables have arbitrary initial values.

In a step of a program execution, any statement from the assign-section is selected and executed. A program execution consists of an infinite number of steps. During program execution each statement is selected and executed infinitely often.

The execution of a UNITY program does not terminate in the conventional sense. Instead, a UNITY program can reach a fixed point, i.e., a state where the execution of any statement leaves the state unchanged.

\subsection{UNITY Logic}

In UNITY there are three logical binary operators, unless, ensures and $\mapsto$ ("leadsto"), to write problem specifications of a program. The operator unless is used to describe safety properties of a program. Safety properties ensure that certain incorrect state transitions never occur. In other words, a correct program will never violate its safety properties. The other logical operators are used to describe progress properties of a program. A progress property is used to describe that a certain predicate will hold at some point in the future.

In [San91], it is shown that the standard definitions of the operators unless, ensures and $\mapsto$ (as given in [ChM88]) in combination with the substitution axiom (, i.e., if $(x=y)$ is an invariant of a program, then $x$ can be replaced by $y$ in all properties of the program") gives an unsound proof system. Modifications to the UNITY operators are proposed that result in a sound and relative complete proof system. An important aspect of the proposed definitions is that, for a single program, the theorems, derived for the standard UNITY operators, still hold for the newly defined operators.

In this section the definitions of the UNITY operators, as proposed by [San91], are given. First, the predicate transformer sst is defined. Next, sst is used to give the definitions of the UNITY operators. The symbols $p, q$ and $r$ denote predicates. 


\subsection{1. sst}

For a given program $F$, sst.F.p (strongest "stable" predicate) is defined as:

sst. $F . p=$

strongest solution of $X:\langle$ for all statements $s$ of $F::\{X\} s\{X\}\rangle \wedge[p \Rightarrow X]$

Let F.INIT denote a predicate that characterises the allowed initial states of a given program $F$. Then, sst.F.(F.INIT) is the strongest invariant of program $F$ and corresponds to the set of all reachable states. Note, in the following sst.F.INIT is used to indicate sst.F.(F.INIT).

\subsection{2. unless}

For a given program $F, p$ unless $q$ is formally defined as

$p$ unless $q=$

sst.F.INIT $\Rightarrow$ for all statements $s$ of $F::\{p \wedge \neg q\} s\{p \vee q\}\rangle$

i.e., if $p$ is true at some point in the execution of the program and $q$ is not, in the next step of the execution $p$ remains true or $q$ becomes true.

Two important special cases of unless, stable and invariant, are given next. The stable-property is, for a given program, formally defined as

stable $p=p$ unless false

i.e., once $p$ becomes true during the program execution, it remains true. Note, however, that $p$ not necessarily ever will hold.

The definition of the invariant-property, given some program $F$, is

$p$ is invariant $=((F . I N I T \Rightarrow p) \wedge$ stable $p)$

i.e., an invariant holds in every state during any execution.

\subsection{3. ensures}

The operator ensures is, for a given program $F$, defined as

$p$ ensures $q=$

$p$ unless $q \wedge\langle$ there is a statement $s$ of $F::\{$ sst.F.INIT $\wedge p \wedge \neg q\} s\{q\}\rangle$

i.e., once $p$ is true during the execution of the program, it remains true as long as $q$ is false, and eventually $q$ becomes true.

\subsection{4. leads-to}

For a given program, $p \mapsto q$, pronounced: $p$ leads to $q$, holds if and only if it can be derived using a finite number of applications of the following rules:

- $(p$ ensures $q) \Rightarrow(p \mapsto q)$

- $((p \mapsto q) \wedge(q \mapsto r)) \Rightarrow(p \mapsto r)$

- For any set $W$, $\langle\forall m: m \in W:: p(m) \mapsto q\rangle \Rightarrow(\langle\exists m: m \in W \quad:: p(m)\rangle \mapsto q)$.

In other words, $p \mapsto q$ holds if and only if once $p$ becomes true during the program execution, $q$ is or will become true eventually. 


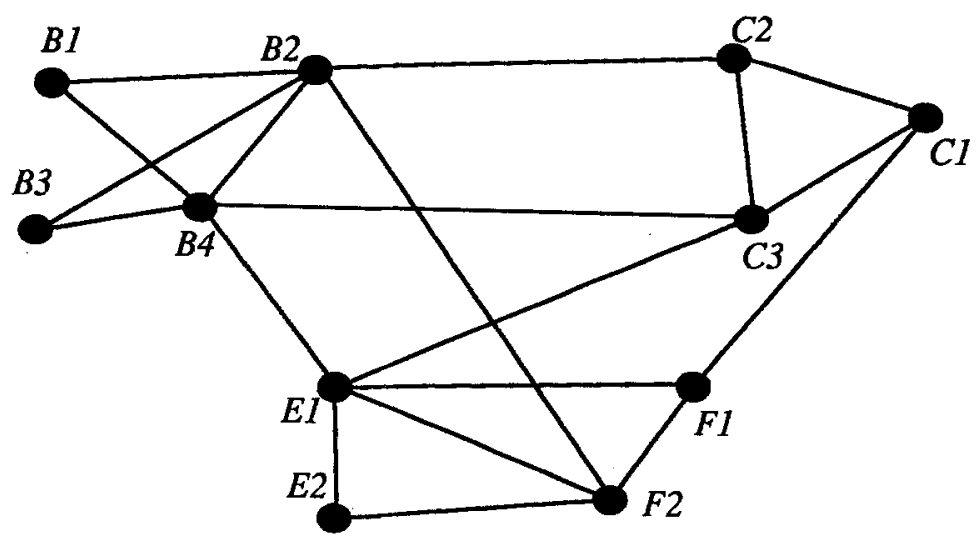

Fig. 1. The network graph.

\section{Hierarchically Divided Networks}

In this article we describe a distributed algorithm on a network that is identified with a bidirectional graph, the network graph $(N, E)$, with edges $E$ and nodes $N$. An edge in $E$ between nodes $a$ and $b$ is denoted by $\{a, b\}$. Nodes $a$ and $b$ are called neighbours if and only if $\{a, b\} \in E$. In fig. 1 an example network graph is given. The nodes of this graph represent processors in the network, the edges represent communication links between them. Every node has local computing capacity and memory to which no other node has (direct) access. Nodes cooperate by exchanging messages over the links connecting them. These links are assumed to be fault-free and have a FIFO behaviour.

Upon the network graph a hierarchical structure is imposed [LUS89]: The network graph is a domain, divided in several subdomains, which in turn are divided in smaller domains, etc. In this view, the smallest possible domains are the nodes of the network graph. The network graph, thus divided, is called a hierarchically divided graph. In fig. 2, a hierarchically divided graph is presented by (arbitrarily) hierarchically dividing the graph of fig. 1. The division of the graph is modeled as a tree, called the division tree. The network domain (the domain comprising the whole network) corresponds to the root of this tree. Subdomains of a domain correspond to sons of the tree-node corresponding to the domain. The leaves of the division tree correspond to the nodes of the network graph. In fig. 3 the division tree of the hierarchically divided graph of fig. 2 is given.

The following convention will be adopted in this article. Nodes in the network graph are denoted by small letters. Capital letters are used to denote arbitrary tree-nodes and domains.

Because of the correspondence between domains and tree-nodes, the following definitions are given in terms of domains as well as tree-nodes.

When tree-node $A$ in the division tree is a son of tree-node $B$ (or $B$ is the father of $A$ ), we write: $A \triangleleft B$ or $B=f(A)$. Analogously, when domain $A$ in the hierarchically divided network is a subdomain of domain $B$, we write: $A \triangleleft B$ or $B=f(A)$. $A$ is contained in $B, A \in B$, or $B$ is an ancestor of $A$, if and only if $B \triangleleft^{*} A$. The notation $f(A) \backslash A$ is used to denote the set $\left\{A^{\prime} \mid A^{\prime} \triangleleft f(A) \wedge A^{\prime} \neq A\right\}$. $A$ is visible to $B$ if $B \in f(A)$, and $B \notin A$. 


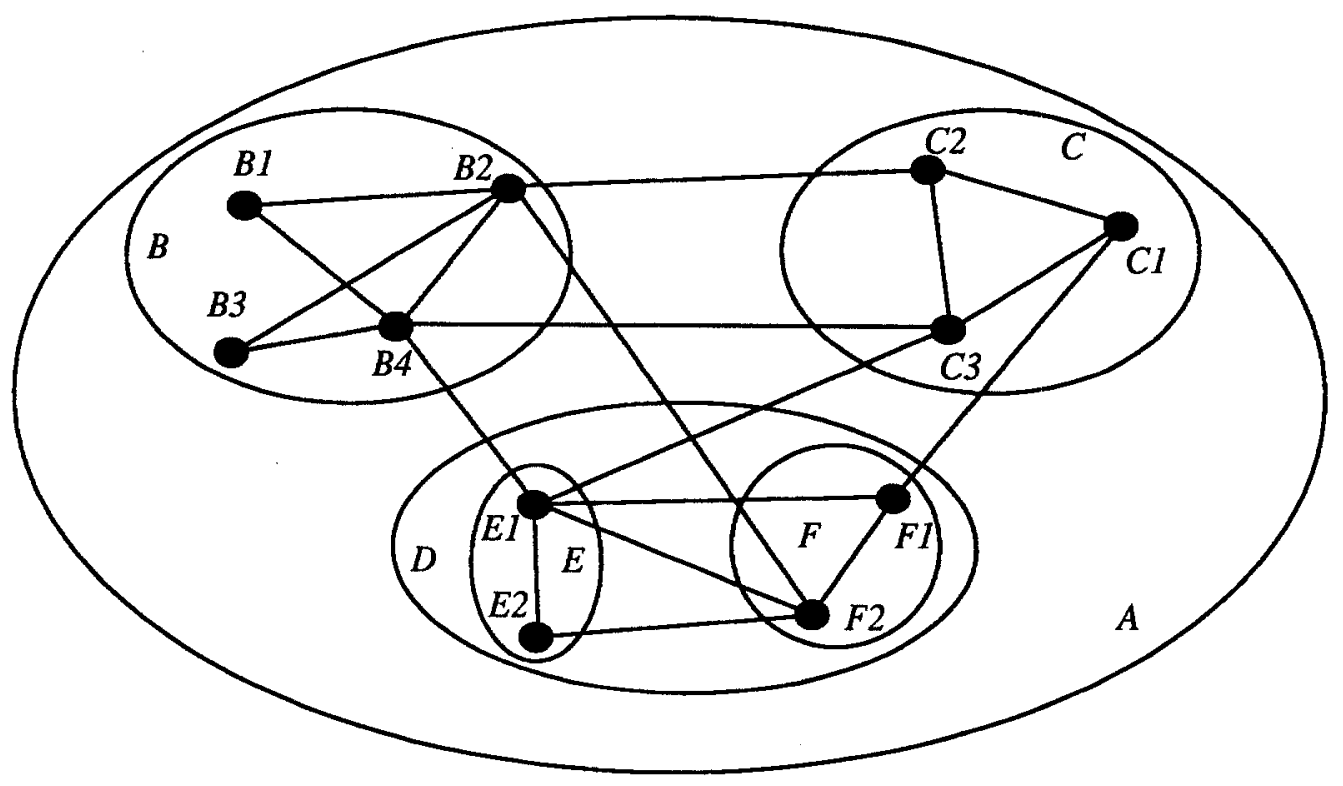

Fig. 2. The hierarchically divided graph.

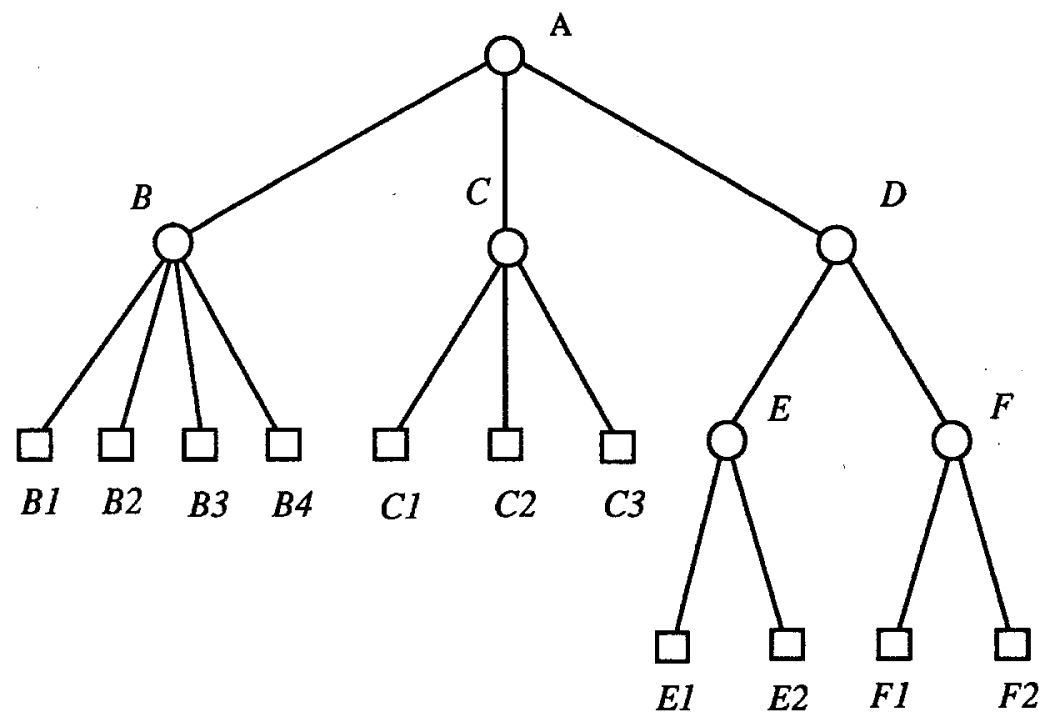

Fig. 3. The division tree. 
As an example, in the hierarchically divided network of fig. 2 , the following relations hold: $B 1 \in B$ and $B 1 \in A, C \triangleleft A, E=D \backslash F$ and $E 2, F, C$ and $B$ are visible to $E 1$.

Note that, by definition, if a domain $A$ is visible to domain $B$, then $A$ is visible to every node in $B$. On the other hand, subdomains of a domain that is visible to a node are not visible to that node. As a consequence, internal details of a domain are hidden from domains, and thus from nodes, outside that domain. This will result in a reduction of the storage complexity of the algorithms to be developed: only a single item of information has to be stored at a node $n$ for each domain visible to $n$, and not for every single node contained in it.

On the hierarchical division we impose the following restriction: every domain is connected. In other words: a message routed between two nodes does not have to leave a common domain of those nodes. As described in [LUS89], subdomains may be shared between domains, i.e., domains may be overlapping. In the following, without loss of generality, it is assumed that each hierarchical division can be modeled as a division tree.

\section{Problem}

In this section we describe the central problem of this article. It is assumed that every node in a hierarchically divided network has an associated data item, called its input. Inputs are not necessarily unique elements from a totally ordered set. The input of a node may change spontaneously and, as a result the maximum of the inputs of all nodes may change. Within finite time after the last change, the global maximum must have been obtained.

\subsection{Formal Description}

Before the specification of the problem is given in UNITY, some additional notation is introduced.

\subsubsection{Notation}

- $G=(N, E)$ denotes the finite graph corresponding to a (hierarchically divided) network.

- $\forall n \in N: \operatorname{inp}(n)$ contains the input of node $n$.

- Variable $m(G)$ is used to hold the maximum of the inputs.

- $\left[a_{1}, a_{2}, \ldots, a_{n}\right]$ denotes the bag, or multiset, of elements $a_{1}, a_{2}, \ldots, a_{n}$. If $n_{1}, \ldots, n_{x}$ are the nodes of $G$, then $\operatorname{nodesval}(G)=\left[\operatorname{inp}\left(n_{1}\right), \operatorname{inp}\left(n_{2}\right), \ldots, \operatorname{inp}\left(n_{x}\right)\right]$.

- $\vec{M}$ denotes a bag of integers.

- When the algorithm has terminated with value $v$, i.e., the algorithm has reached a fixed point and the computed maximum equals $v$, we say that $\operatorname{term}(G, v)$ holds. Then, the variable $m(G)$ equals the maximum $v$ of all inputs, i.e.,

$$
\operatorname{term}(G, v) \Rightarrow m(G)=v=\langle\max n: n \in N \quad: \operatorname{inp}(n)\rangle .
$$




\subsubsection{Specification}

The specification of maintaining and obtaining the maximum value of the inputs in a network $G$, where each node has an input, can be formulated in two rules. The first rule states that once the algorithm has terminated with a value $v$ which equals the maximum of the inputs, i.e., $\operatorname{term}(G, v)$ holds, it remains terminated with value $v$ until an input has changed. Formally,

SP1: $(\operatorname{term}(G, v) \wedge$ nodesval $(G)=\vec{M})$ unless $($ nodesval $(G) \neq \vec{M})$

The second rule states that, given a bag of inputs, eventually the algorithm terminates or the bag of inputs changes. Formally,

$$
\mathrm{SP} 2:(\operatorname{nodesval}(G)=\vec{M}) \mapsto(\langle\exists v \quad:: \operatorname{term}(G, v)\rangle \vee \operatorname{nodesval}(G) \neq \vec{M})
$$

\section{Update Algorithm on a Division Tree}

In this section we present an update algorithm that operates on a division tree after the input of a leaf has changed. In this algorithm, leaves as well as internal tree-nodes of the division tree are assumed to be capable of performing actions and of storing values (of variables). Keep in mind, however, that in a physical network, nodes are the only entities having these properties. Since tree-nodes in the division tree model domains in the hierarchical division of the network, the actions of the domains have to be simulated by the physical nodes "on behalf of the domains."

The following questions, then, have to be answered. Which nodes simulate the actions corresponding to a domain? Do we need special nodes, or can the effect of the algorithm executed by a domain be established by all nodes contained in it?

For every tree-node $D$ in the division tree the following variables are maintained:

$m^{D}(D)$ is $D$ 's current view of the maximum of the inputs under $D$.

$m^{D}(C)$ is $D$ 's current view of $m^{C}(C)$ for every $C \triangleleft D$, if $D$ is not a leaf.

In a stable state, the following relations hold for every tree-node $D$ and $C \triangleleft D$.

$$
\begin{array}{ll}
m^{D}(D) & = \begin{cases}\operatorname{inp}(D) & \text { if } D \text { is a leaf } \\
\max \left\{m^{D}(C) \mid C \triangleleft D\right\} & \text { otherwise }\end{cases} \\
m^{D}(C)=m^{C}(C) &
\end{array}
$$

Let $R$ be the root of the division tree corresponding to the hierarchical division on $G=(N, E)$. Then, the following relation holds in a stable state:

$$
m^{R}(R)=\max \{\operatorname{inp}(n) \mid n \in N\}
$$

In Section 5.1 the update algorithm is informally described. In Section 5.2 a formal description in UNITY is given. The formal description consists of a specification of the solution strategy, a correctness proof of this solution strategy and a program satisfying the solution strategy. In Section 5.3 the informally and formally described algorithms are compared. 


\subsection{Informal Description}

We now describe the distributed algorithm that is performed as a result of a change in the input of a leaf. Relations (1) and (2) suggest the following update algorithm. Upon detecting a change in $\operatorname{inp}(D)$, leaf $D$ updates $m^{D}(D)$ and reports the value to its father. A tree-node $D$, notified of a change in $m^{C}(C)$ with $C \triangleleft D$, updates $m^{D}(C)$, recomputes $m^{D}(D)$ and, if it has changed, reports this to its father (unless $D$ is the root).

The resulting algorithm is presented slightly more formally below. It is assumed that the datastructure is correctly initialised, i.e., relations 1 and 2 are satisfied (e.g., initially, all variables and inputs contain $-\infty$ ).

\section{Algorithm I (division tree)}

- Upon detecting a change in $\operatorname{inp}(D)$, leaf $D$ updates $m^{D}(D)$ and sends $U P D\left(D, m^{D}(D)\right)$ to $f(D)$.

- Upon receipt of $U P D\left(C, m_{C}\right)$, tree-node $D$ performs the following actions:

$m^{D}(C):=m_{C}$;

$m^{D}(D):=\max \left\{m^{D}(C) \mid C \triangleleft D\right\} ;$

if $m^{D}(D)$ changed and $D$ is not the root

then send $U P D\left(D, m^{D}(D)\right)$ to $f(D)$

fi

\subsection{Formal Description}

Before the specification of the algorithm on a division tree is given, first some more notation is introduced.

\subsubsection{Notation}

For every tree-node $D$ :

- The set leaves $(D)$ contains $D$ itself if $D$ is a leaf or else the leaves under tree-node $D$.

- Let + be the bag-union. Then leavesval $(D)$ is defined as follows:

leavesval $(D)=[\operatorname{inp}(D)]$, if leaves $(D)=\{D\}$.

Otherwise, let $C_{1}, \ldots, C_{x}$ be the sons of $D$, then

leavesval $(D)=$ leavesval $\left(C_{1}\right)+$ leavesval $\left(C_{2}\right)+\ldots+$ leavesval $\left(C_{x}\right)$.

Note: leavesval $(R)=$ nodesval $(G)$, if and only if $R$ is the root of the division tree corresponding to the hierarchical division on $G$.

- The value of tree-node $D$ must be reported to its father. Let fatherknows $(D, v)$ be defined by:

$$
\text { fatherknows }(D, v)=\left(\left(m^{f(D)}(D)=v\right) \vee(D=R)\right)
$$

where $R$ is the root of the division tree.

- The algorithm has finished for tree-node $D$ with value $v$ if and only if the variable $m^{D}(D)$ contains $v$ that is the maximum of all the values of its sons, and the algorithm has terminated (which is defined next) for these sons. 


$$
\begin{aligned}
& \text { hfinished }(D, v)= \\
& \qquad \begin{aligned}
v=m^{D}(D)=\operatorname{inp}(D) & \text { if leaves }(D)=\{D\} \\
v=m^{D}(D)=\left\langle\max C: C \triangleleft D:: m^{D}(C)\right\rangle \wedge & \\
\left\langle\forall C: C \triangleleft D:: \text { hterm }\left(C, m^{C}(C)\right)\right\rangle & \text { otherwise. }
\end{aligned}
\end{aligned}
$$

- The algorithm has terminated with value $v$ for tree-node $D$ if and only if the algorithm has finished with value $v$ for $D$ and this value of $D$ is known by the father of $D$.

$$
\operatorname{hterm}(D, v)=(\text { hinished }(D, v) \wedge \text { fatherknows }(D, v)) .
$$

\subsubsection{The Solution Strategy: Formal Description}

We derive a formal specification from the informal description of an algorithm on the division tree as given in Section 5.1. First, two safety properties are derived.

After tree-node $D$ and every tree-node under $D$ has computed the maximum of its underlying inputs, i.e., $\langle\exists v::$ hfinished $(D, v)\rangle$, no tree-node under $D$ nor $D$ itself is allowed to change its computed maximum unless an input under $D$ has changed. This restriction suggests the following condition:

$$
\text { SP3: }(\text { hinished }(D, v) \wedge \text { leavesval }(D)=\vec{M}) \text { unless }(\text { leavesval }(D) \neq \vec{M})
$$

When tree-node $D$ has computed the maximum value of its underlying inputs, the father-tree-node knows this maximum value and every tree-node under $D$ has computed the maximum value of its underlying inputs, i.e., when $h \operatorname{term}(D, v)$ holds, then the father-tree-node is not allowed to change its value of $D$ unless $D$ or a tree-node under $D$ recomputes its maximum value (because of a change in an input), i.e., $\neg$ hinished $(D, v)$. This gives the following condition:

SP4: hterm $(D, v)$ unless $\rightarrow$ hfinished $(D, v)$

Next, two progress conditions are derived. When every tree-node under treenode $D$ has computed the maximum of its underlying inputs, and $D$ is aware of the value of each son, i.e., $\left\langle\forall C: C \triangleleft D:: h \operatorname{term}\left(C, v_{c}\right)\right\rangle$ holds, tree-node $D$ can compute the maximum value of its underlying inputs. After recomputation two cases might have occurred. The first case is that after the computation $D$ has the maximum of the underlying inputs, i.e., $\langle\exists v::$ hfinished $(D, v)\rangle$. The second case is that, during the computation, an input under $D$ has changed, in other words, not every tree-node under $D$ is aware of the maximum of its underlying inputs, i.e., $\left\langle\exists C: C \triangleleft D:: \neg\right.$ hfinished $\left.\left.\left(C, v_{c}\right)\right\rangle\right)$. Thus,

$$
\begin{aligned}
\mathrm{SP} 5:\langle\forall C: & \left.: C \triangleleft D:: \text { hterm }\left(C, v_{c}\right)\right\rangle \mapsto \\
& \left(\langle\exists v:: \text { hfinished }(D, v)\rangle \vee\left\langle\exists C: C \triangleleft D:: \neg \text { hinished }\left(C, v_{c}\right)\right\rangle\right)
\end{aligned}
$$

The last (progress) condition is rather straightforward. After tree-node $D$, and every tree-node under $D$, has computed its maximum value of its underlying inputs ( $h$ finished $(D, v)$ holds), the father-tree-node of $D$ must know within finite time the maximum value of the inputs under $D$ (which equals $v$ ) or not every tree-node under $D$ knows the maximum value of its underlying inputs anymore ( $\neg$ hinished $(D, v)$ holds). Thus,

SP6: hfinished $(D, v) \mapsto(\operatorname{hterm}(D, v) \vee \neg$ hfinished $(D, v))$

The specification of a solution strategy for obtaining and maintaining the 
maximum value of the inputs at the leaves under a node $D$ in a division tree, consists of SP3 through SP6.

\subsubsection{Proof of Correctness of the Solution Strategy}

To prove the correctness of the solution strategy, we prove that the problem specification (SP1 and SP2) is satisfied by any strategy satisfying SP3 through SP6. The formal proof is given in appendix B, here, we give an outline of the method used.

To prove that SP1 is met, it suffices to prove that if the algorithm has terminated for tree-node $D$ with value $v$, then $m^{D}(D)=v$ equals the maximum of the inputs under $D$. In that case, if $R$ is the root of the division tree corresponding to the hierarchical division on the graph $G$ and $h \operatorname{term}(R, v)$ holds, then $m^{R}(R)=v$ equals the maximum of all inputs in the graph $G$. Thus, if $h \operatorname{term}(R, v)$ holds, $\operatorname{term}(G, v)$ holds as well, and vice versa.

The proof that if hterm $(D, v)$ holds, $m^{D}(D)=v$ equals the maximum of the inputs under $D$, is done by induction on the structure of the division tree. For every leaf $D$ of the division tree it directly follows that when $h$ term $(D, v)$ holds, $m^{D}(D)=v=\operatorname{inp}(D)$. Next, given that hterm $(D, v)$ holds, thus also hterm $\left(C, m^{C}(C)\right)$, it follows from the induction hypothesis that $m^{C}(C)$ equals the maximum of the inputs under tree-node $C$, for every $C \triangleleft D$, and it can be proven that $m^{D}(D)$ equals the maximum of the inputs under $D$.

To prove that SP2 is met, we have to verify that always within finite time an algorithm, satisfying SP3, SP4, SP5 and SP6, terminates for the root or at least one input has changed. Since $h \operatorname{term}(R, v)=h$ finished $(R, v)$ for the root $R$, it is sufficient to prove for every tree-node $D$ that always within finite time the algorithm will finish for $D$ or at least one input under $D$ has been changed.

The proof is given in stages. First, it is proven that if the algorithm has finished for the sons of a tree-node, the algorithm will terminate within finite time for these sons or at least one input under such a son has changed. Next, it has been proven that if the algorithm has terminated for the sons of a tree-node, the algorithm will finish within finite time for the tree-node or at least one input under the tree-node has changed. Finally, these results have been combined (using an induction argument) proving that always within finite time the algorithm will finish for some tree-node or an input under this tree-node has changed.

\subsubsection{The Solution Strategy: Formal Description Refined}

The given solution strategy is not yet in a form that can be directly translated to a program. In order to come up with such a specification, the progress conditions SP5 and SP6 must be refined, because leads-to properties cannot be directly transformed into program text.

SP5 states that if the algorithm has terminated for every son of tree-node $D$, $m^{D}(D)$ must be recomputed such that at some point in the future the algorithm has finished for $D$ or the algorithm has not finished anymore for at least one subdomain of $D$ (due to a change of an input under $D$ ). By ensuring that always at some point in the future $m^{D}(D)$ is recomputed, this property is satisfied. This suggests the following relations:

$$
\text { SP7: (leaves }(D)=\{D\}) \text { ensures }\left(m^{D}(D)=\operatorname{inp}(D)\right)
$$


SP8: $($ leaves $(D) \neq\{D\})$ ensures $\left(m^{D}(D)=\left\langle\max C: C \triangleleft D:: m^{D}(C)\right\rangle\right)$

SP6 states that if the algorithm has finished for a tree-node $D$, within finite time the father-tree-node $f(D)$ of $D$ is aware of $m^{D}(D)$, i.e., $m^{f(D)}(D)=m^{D}(D)$. By ensuring that always at some moment in the future $m^{f(D)}(D)$ is updated to $m^{D}(D)$, this property is satisfied. This suggests the following relation:

SP9: True ensures fatherknows $\left(D, m^{D}(D)\right)$

The (refined) specification of the solution strategy for obtaining and maintaining the maximum value of the inputs at the leaves consists of SP3, SP4, SP7, SP8 and SP9.

A formal proof of this refinement step is given in Appendix C.

\subsubsection{Derivation of a Program from the Specification}

A program follows directly from the specification of the solution strategy. The function $\operatorname{inp}(D)$ delivers the input of some leaf $D$.

SP7 suggests the recomputation of $m^{D}(D)$ when $D$ is a leaf, i.e.,

$$
\left\langle\square D: D \in \text { leaves }(D):: m^{D}(D):=\operatorname{inp}(D)\right\rangle
$$

SP8 suggests the recomputation of $m^{D}(D)$ when $D$ is not a leaf, i.e.,

$$
\left\langle\square D: \neg(D \in \text { leaves }(D)):: m^{D}(D):=\left\langle\max C: C \triangleleft D:: m^{D}(C)\right\rangle\right\rangle \text {. }
$$

SP9 suggests that every tree-node $D$ is aware of $m^{C}(C)$, with $C \triangleleft D$, i.e.,

$$
\left\langle\square C, D: C \triangleleft D:: m^{D}(C):=m^{C}(C)\right\rangle \text {. }
$$

To show that these statements do not violate the unless-relations SP3 and SP4 and the unless-parts of SP7, SP8 and SP9 is rather straightforward and left to the reader.

The complete program follows.

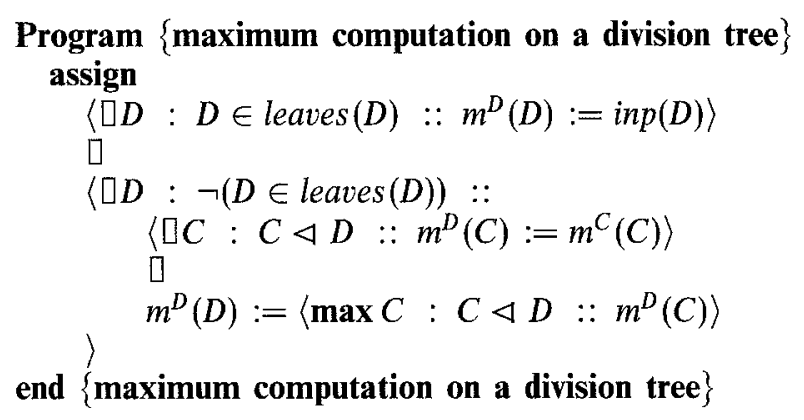

\subsection{Comparison}

In this section, the program in Section 5.1 informally described and the UNITY program of Section 5.2.5 are compared. We will show that by refining the UNITY program, the resulting program is equivalent to the informally described program.

The informal algorithm of Section 5.1, is described using message-passing 
processes. In the given UNITY program processes communicate using shared variables. Every variable $m^{D}(D)$ is shared between processes, i.e., within one process $m^{D}(D)$ is assigned a value, which is computed using local variables (inp $(D)$ or $m^{D}(C)$ with $\left.C \triangleleft D\right)$, and within another process $m^{D}(D)$ is assigned to $m^{f(D)}(D)$. This can be implemented by communications between the processes, as follows: the first process sends the value of $m^{D}(D)$ to the other process which receives the value and assigns it to $m^{f(D)}(D)$ (as is done in the informally described algorithm).

In UNITY a first-in-first-out channel can be represented as a variable whose value is a sequence of messages. A message can be sent by appending it to the end of the sequence. By removing the head of the sequence, a message can be received.

In the following UNITY program directed channels, from each tree-node $D$ (if $D$ is not the root) to its father, are implemented as a sequence of integers, denoted as $(D, f(D))$.c. The concatenation operator for sequences is '; $;$. Initially, no sequence has an item. This is represented by the sequence having item null. Sending an integer $m$ on channel $(D, f(D)) . c$ is done with the following statement:

$$
(D, f(D)) . c:=(D, f(D)) . c ; m
$$

Receiving a message from channel $(D, f(D)) . c$ in variable $m$ is done by executing the following statement:

$$
m,(D, f(D)) \cdot c:=\operatorname{head}((D, f(D)) \cdot c), \operatorname{tail}((D, f(D) . c)
$$

The UNITY program from Section 5.2.5 describes what should be done, i.e., it specifies the state transformations. However, the order in which the statements should be executed is not prescribed. The only requirement imposed on the execution is that each assignment be executed infinitely often.

The informal program from Section 5.1 is more deterministic. For example, after a tree-node is notified of a change in the value of a son, the maximum of the tree-node is recomputed. In the UNITY program, a tree-node may be notified of a number of changes (of its sons) before the maximum value of the tree-node is recomputed.

By introducing boolean variables the UNITY program can be restricted to simulate the execution of the informal algorithm. The boolean variables specify when the execution of a statement must have any effect, i.e., when the execution of a statement should result in a state change. In the following UNITY program, boolean arrays notified and changed, both indexed by the tree-nodes, are used. The value of notified $[D]$ is true if and only if tree-node $D$ is notified of a change in the value of a son and $m^{D}(D)$ is not yet recomputed. The value of changed $[D]$ is true if and only if the father of $D$ (if present) has not yet been notified of the latest change in $m^{D}(D)$.

Since leafs do not have sons, notified $[D]$ is not necessary for leaf $D$. Moreover, if a leaf $D$ is aware of a change in $\operatorname{inp}(D)$, the new value of $m^{D}(D)$ (which equals inp $(D)$ ). can be immediately sent to $f(D)$ without first recomputing it. Therefore, leaf $D$ does not use changed $[D]$ either. 


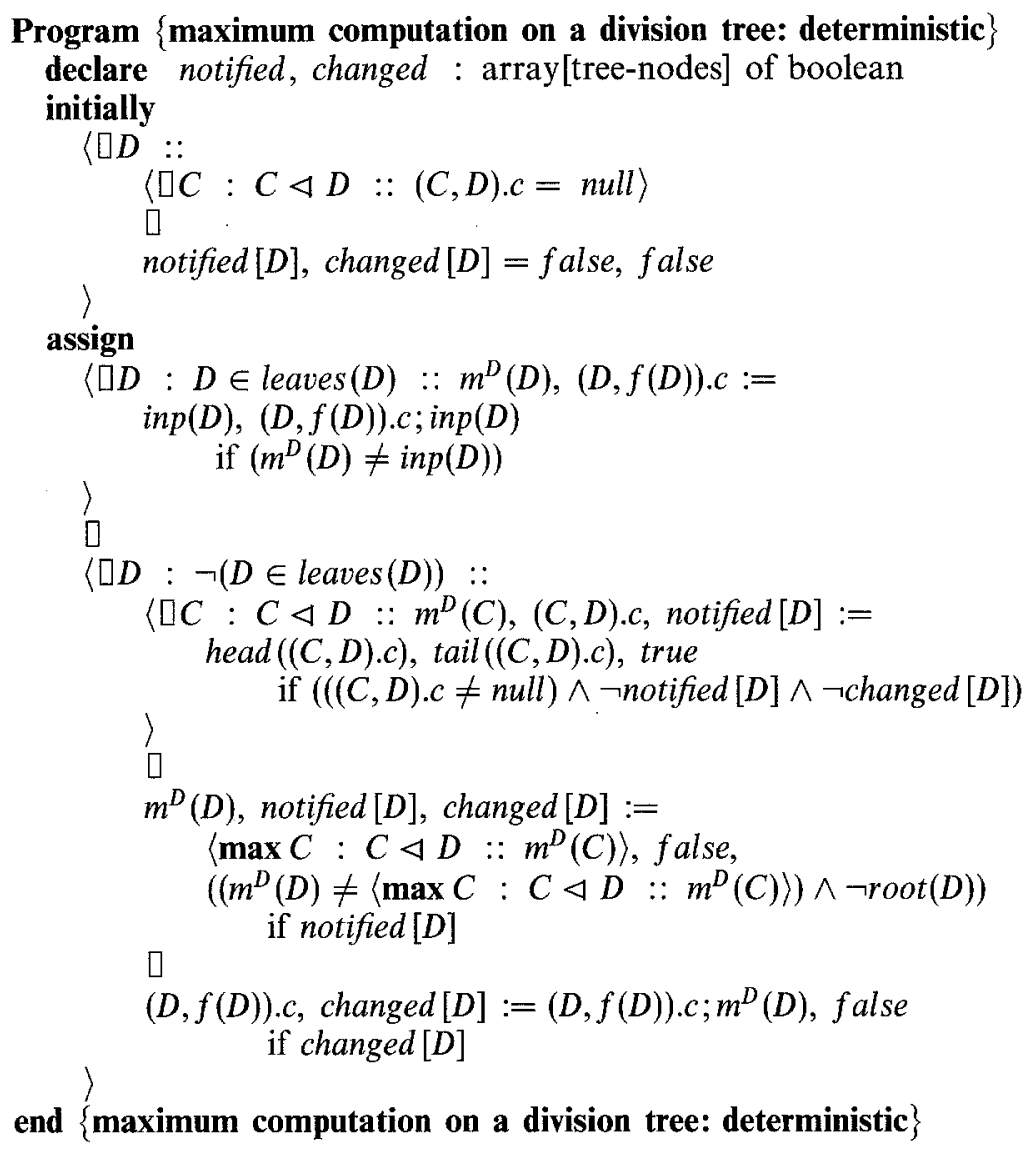

\section{Update Algorithm on a Hierarchically Divided Graph}

In this section we will distribute the algorithm on a division tree as described in Section 5. A domain (a tree-node in the division tree) is simulated by the cooperation of all nodes contained in it. For that purpose we distribute the information kept by any tree-node $D$ in the division tree in Algorithm I over the nodes contained in domain $D$ in the hierarchically divided network. This is realised by a "projection" in the division tree of all information onto every leaf in the subtree below the tree-node containing the information. As a result, every node keeps the variables of its superdomains which were maintained by the corresponding tree-nodes in the update algorithm on a division tree. Thus, for every domain $D$, node $n \in D$ maintains the following variables:

$m_{n}^{D}(D)$ is $\operatorname{inp}(D)$ if $D$ is a node, and $n$ 's view of the maximum of the inputs contained in $D$, if $D$ is not a node.

$m_{n}^{D}(C)$ is $n$ 's view of the maximum of the inputs contained in $C$, for every $C \triangleleft D$, if $D$ is not a node. 
In a stable state, the following relations hold for every $n, n^{\prime} \in D$ and $C \triangleleft D$ :

$$
\begin{aligned}
& m_{n}^{D}(D)= \begin{cases}\operatorname{inp}(D) & \text { if } n=D \\
\max \left\{m_{n}^{D}(C) \mid C \triangleleft D\right\} & \text { otherwise }\end{cases} \\
& m_{n}^{D}(C)=m_{n}^{C}(C) \quad \text { if } n \in C \\
& m_{n}^{D}(C)=m_{n^{\prime}}^{D}(C)
\end{aligned}
$$

Let $G=(N, E), R$ be the network domain and $n \in R$. Then, the following relation holds, when, eventually, the variables are correctly filled in:

$$
m_{n}^{R}(R)=\max \{\operatorname{inp}(n) \mid n \in N\}
$$

\subsection{Informal Description}

In this section an algorithm is derived to obtain and maintain the maximum value of a frequently changing bag of inputs in a hierarchically divided network. The algorithm is derived in two stages. First, an algorithm is given to obtain the maximum value after one input has changed. Next, this algorithm is extended to deal with the case multiple changes of inputs occur at the same time.

In Section 6.2 a formal specification of the resulting algorithm, together with a correctness proof are presented.

\subsubsection{Updating After a Single Change}

Below, we describe how the update algorithm that was presented in the previous section as an abstract algorithm on a division tree can be implemented as a distributed algorithm. In this implementation, the nodes are the only entities capable of performing actions. Thus, the actions of a domain as prescribed by the abstract algorithm will be performed by the nodes contained in the domain.

When, in the abstract algorithm, an increased input of a node results in the increase of the maximum value of a number of ancestors, this effect was realised in subsequent steps in which a domain (tree-node) passed its new maximum to its parent. This communication of information up into the division tree, will, in the distributed algorithm, be implemented by subsequent broadcasts within domains. These broadcasts are carried out, as it were, in a wave carrying the information to nodes contained in a domain, and every time it is concluded that the information must also be reported to the superdomain, the wave front is extended towards the borders of that superdomain.

In the following description, let $m(D)$ denote the maximum of the inputs contained in $D$. When the input $m(n)$ of a node $n$ changes, according to the abstract algorithm, this must be reported to $D$, the superdomain of $n$. In the distributed algorithm, this is implemented by broadcasting the new value of $m(n)$ to all brothers of $n$. The result is that every node contained in $D$ knows the new value of $m(n)$, and can determine whether $m(D)$ has changed. In the algorithm, this is indicated by node $n^{\prime} \in D$ computing $m_{n^{\prime}}^{D}(D):=\max \left\{m_{n^{\prime}}^{D}\left(n_{i}\right) \mid n_{i} \triangleleft D\right\}$.

In the case that $m(D)$ has changed, this must be reported to all brothers of $D$. In other words, the broadcast will be extended within $f(D)$. This extension of the broadcast is initiated by the border nodes of $D$, i.e., nodes with neighbours contained in $f(D) \backslash D$. These border nodes, after having updated their copy of $m(D)$ and (at the same time) having updated $m^{f(D)}(D)$ to this value, start broadcasting 
this new value within $f(D) \backslash D$. This process of broadening the broadcast continues until the smallest domain $A$ of which the maximum does not change has been reached. Border nodes of this domain will conclude that brothers of $A$ do not have to change their estimate of $m(A)$, and they, thus, will not extend the broadcast.

The resulting algorithm is presented below.

\section{Algorithm II (network, single change)}

- Upon detecting a change in $\operatorname{inp}(n)$, node $n$ performs $\operatorname{Accept}_{n}(n, \operatorname{inp}(n))$.

- Upon receipt of $U P D\left(D, m_{D}\right)$, node $n$ performs the following actions:

if $m_{n}^{f(D)}(D) \neq m_{D}$

then $m_{n}^{f(D)}(D):=m_{D}$;

Broadcast $_{n}\left(D, m_{D}\right)$;

if $m_{n}^{f(D)}(f(D)) \neq \max \left\{m_{n}^{f(D)}\left(D_{i}\right) \mid D_{i} \triangleleft f(D)\right\}$

then $\operatorname{Accept}_{n}\left(f(D), \max \left\{m_{n}^{f(D)}\left(D_{i}\right) \mid D_{i} \triangleleft f(D)\right\}\right)$

fi

fi,

where

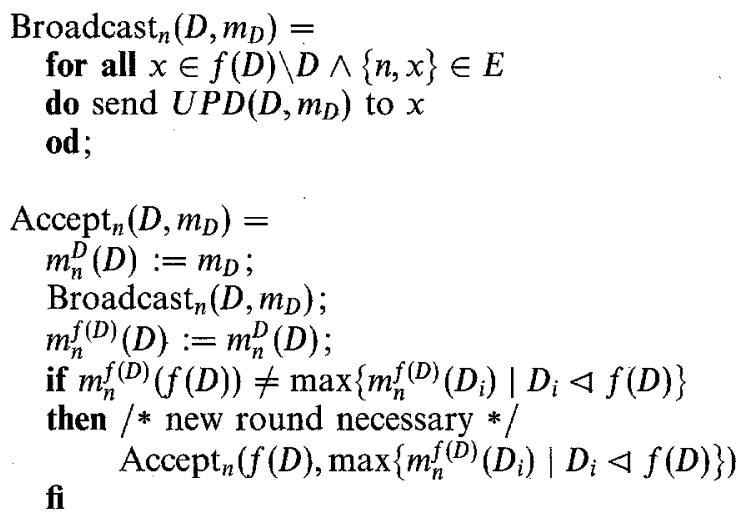

Note, that as a consequence of a single input change, a node accepts any information (about the maximum value of the visible domain containing the node whose input has changed) at most once, and, thus, sends this information at most once over its adjoining links. In the worst case, two neighbour nodes, after accepting the same information, send each other this information. Thus, the algorithm requires at most two update messages to be sent over every link in the network. Moreover, the update messages are confined to the largest domain with a subdomain of which the input changes.

\subsubsection{Updating After Multiple Changes}

In this section we extend Algorithm II to deal with the case of multiple changes of inputs occurring at the same time. When the input of a node changes, the node starts reporting this change using Algorithm II. It is possible, however, that during this process the input of another node changes, too. Since the information 
about this change will not immediately reach the first node, this node may start a broadcast based on out-of-date information. Therefore, update messages reporting different values of the same domain may be in transit at the same time.

As before, in order to simulate the actions of a domain by the cooperation of the nodes contained in it, every node contained in the domain maintains the maximum value of the inputs contained in each of its subdomains. Because of this projection of all information in the division tree onto every leaf, nodes are not aware of the internal structure of their visible domains. Specifically, nodes do not keep the maximum value of the inputs contained in the subdomains of their visible domains. Nodes, therefore, cannot determine whether the information received from a visible domain is up-to-date or not (i.e., whether the value equals the current maximum of the inputs contained in the subdomains).

In [Per83], Perlman describes broadcast algorithms of routing information that stabilise in reasonable time without human intervention after any malfunctioning equipment is repaired or disconnected. If each node in the network has a local clock that is guaranteed to be monotonically increasing, each message can be timestamped by the nodes starting the broadcast of the message with the local clock. The local clock scheme requires hardware that is not always available in packet switches.

Another possibility to distinguish between succeeding broadcasts for a domain is the use of sequence numbers. Each node capable of starting a broadcast for a domain (i.e., each border node), maintains a counter, called the sequence number for that domain. Every time such a node starts a broadcast for a domain, the sequence number for that domain is incremented and the message labelled with it. On receiving an update message with information that is more recent than the information currently available, a node accepts this information by executing Algorithm II. A node accepts a message as being more recent, if the sequence number in the message is higher than the one kept until then. Nodes, therefore, maintain sequence numbers for every visible domain. Border nodes also have to keep sequence numbers for the domains of which they are border nodes. In order to keep this information up-to-date, every time an update of this number takes place, a broadcast of it is performed in the domain.

In [Per83] it is described how the sequence number scheme can be extended to deal with the possibility of wrap-around and failures, such as the possibility of long delayed packets, network partitions and hardware failures. Here, we assume that counters are infinite, and that no failures occur.

Two broadcasts started by different border nodes of a domain, even when labeled with the same sequence number, need not have the same value. For example, suppose $n, n^{\prime} \triangleleft D$ and both $\operatorname{inp}(n)$ and $\operatorname{inp}\left(n^{\prime}\right)$ change. As a result of the changes, both $n, n^{\prime}$ broadcast their new input values within $D$ and recompute the maximum value of $D$. In this case, it is possible that both nodes conclude that the maximum value of $D$ has changed (for example, when both inp(n) and $\operatorname{inp}\left(n^{\prime}\right)$ have increased to a value bigger than the maximum value of $D$ before the change), and start broadcasting their computed value for $D$, labeled with the same sequence number (for example, $n$ broadcast $\operatorname{inp}(n)$ for $D$ with sequence number $S$ and $n^{\prime}$ broadcasts $\operatorname{in} p\left(n^{\prime}\right) \neq \operatorname{inp}(n)$ with the same sequence number $S$ ). However, nodes $n$ and $n^{\prime}$ will receive an update message with the new input value of $n^{\prime}$ and $n$ respectively. After the receipt, each node recomputes the maximum value of $D$. At least one node must conclude that the maximum value of $D$ has changed and starts a new broadcast for $D$ labeled with a higher sequence number. (For example, if $\operatorname{inp}\left(n^{\prime}\right)$ has become the maximum value in $D, n$ starts broadcasting 
$\operatorname{inp}\left(n^{\prime}\right)$ for $D$ labeled with the biggest sequence number for $D$. Then, inp(n) will be overwritten by inp $\left(n^{\prime}\right)$ as a maximum value for $D$.)

In general, when two border nodes started a broadcast for a domain $D$ with different values, at least one border node has not yet received at least one update message of a broadcast for a subdomain of $D$ currently in the network. It is guaranteed that this border node will receive this missing information within finite time and start a new broadcast for domain $D$. This new value may be the same value the other border node already broadcast for $D$. The same value may then be accepted twice by some nodes. The algorithm can be adapted to loose this inefficiency. Since optimising our algorithm is beyond the scope of this article, we do not deal with this issue.

We denote by $S_{n}(D)$ the sequence number of a domain $D$ stored at node $n$. Initially, for every node $n$ and domain $D$ with $n \in D, S_{n}(D)$ contains 0 , and for every $C \triangleleft D, m_{n}^{D}(C)$ and $m_{n}^{D}(D)$ contain $-\infty$. The set Border $(D)$ contains the border nodes of $D$. After a border node $n$ of some domain $D$ has updated $m_{n}^{D}(D)$, $S_{n}(D)$ is incremented and $n$ starts a broadcast of $m_{n}^{D}(D)$ labeled with $S_{n}(D)$, i.e., $U P D\left(D, m_{n}^{D}(D), S_{n}(D)\right)$ is sent to all neighbours contained in $f(D)$. Then, all nodes contained in $f(D)$ can update their sequence number for $D$. Moreover, every node $x$ contained in $f(D) \backslash D$ is able to update $m_{n}^{f(D)}(D)$.

Note, that in the algorithm for a single change, as described in Section 6.1.1, a broadcast for a domain $D$ is performed within $f(D) \backslash D$, i.e., $U P D\left(D, m_{D}\right)$ messages are sent to nodes $x \in f(D) \backslash D$. However, in this case, a broadcast for a domain $D$ is performed in $f(D)$, i.e., $U P D\left(D, m_{D}, S_{D}\right)$ messages are also sent to nodes contained in $D$.

\section{Algorithm III (network, multiple changes)}

- Upon detecting a change in $\operatorname{inp}(n)$, node $n$ performs $\operatorname{Accept}_{n}\left(n, \operatorname{inp}(n), S_{n}(n)+1\right)$.

- Upon receipt of $U P D\left(D, m_{D}, S_{D}\right)$, node $n$ performs the following actions:

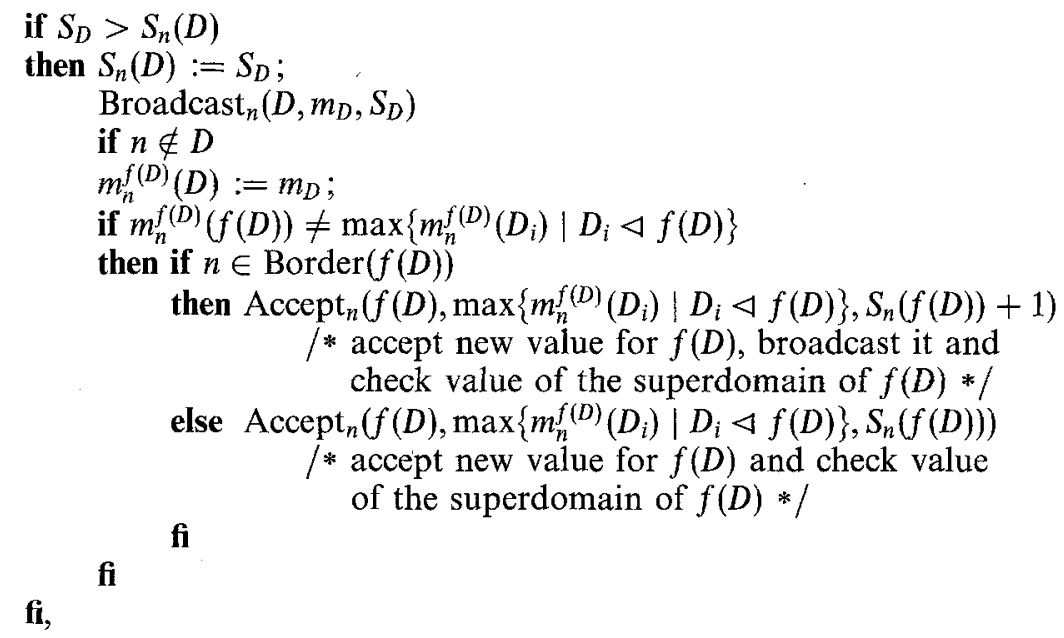

where

$\operatorname{Broadcast}_{n}\left(D, m_{D}, S_{D}\right)=$

for all $\{n, x\}, x \in f(D)$ 


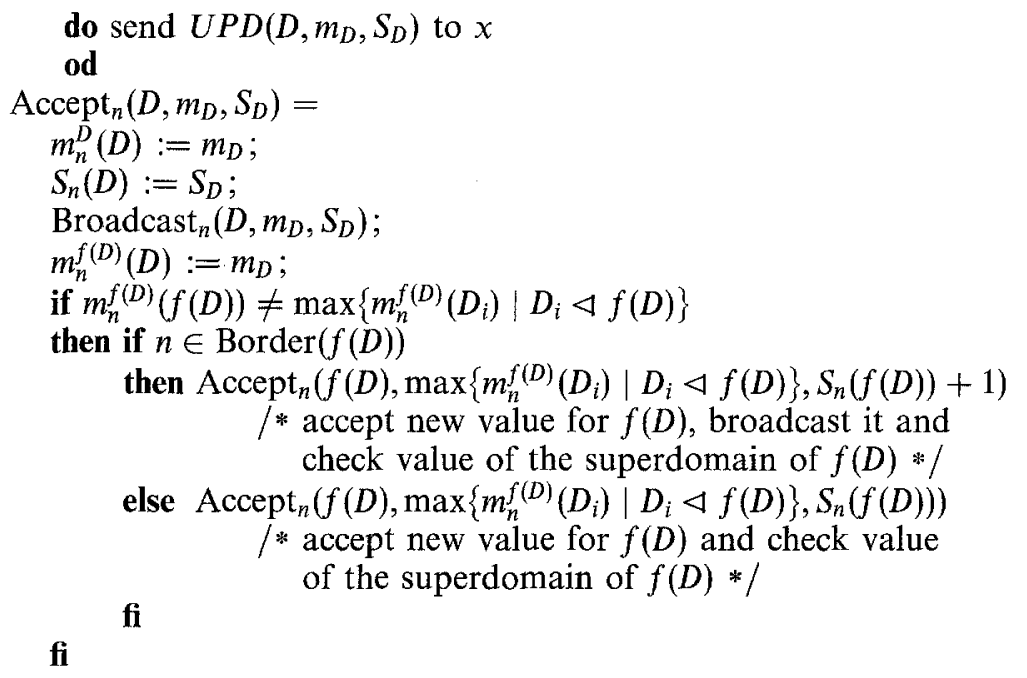

\subsection{Formal Description}

In this section a formal specification, correctness proof and description of the resulting distributed algorithm on a hierarchically divided network, as informally described in Section 6.1.2, are given. The specification is obtained by transforming the specification for the solution strategy on a division tree. The correctness of the solution strategy on the hierarchically divided network then follows from the correctness of the solution strategy on the division tree.

As already stated in the informal description of Section 6.1 , every action by a tree-node in the algorithm on a division tree, will be performed by the cooperation of all nodes contained in the corresponding domain. In order to obtain the specification, first the notation of Section 5.2.1 is adapted to this view. For every domain $D, T D$ denotes the corresponding tree-node.

\subsubsection{Notation}

For every domain $D$ :

- The set Dnodes $(D)$ contains $D$ itself if $D$ is a node or else the nodes contained in domain $D$.

Note that Dnodes $(D)=$ leaves $(T D)$.

- Dnodesval $(D)$ is defined as follows:

Dnodesval $(D)=[\operatorname{inp}(D)]$, if Dnodes $(D)=\{D\}$.

Otherwise, let $C_{1}, \ldots, C_{x}$ be the subdomains of $D$, then

Dnodesval $(D)=$ Dnodesval $\left(C_{1}\right)+$ Dnodesval $\left(C_{2}\right)+\ldots+$ Dnodesval $\left(C_{x}\right)$.

Note that Dnodesval $(D)=$ leavesval $(T D)$.

- In the algorithm on a division tree, the value of a tree-node must be reported to its father. Here, the value must also be reported to its father, i.e., the value must be announced to all nodes contained in the superdomain of the domain. Dfatherknows $(D, v)$ holds if and only if all nodes $n$ contained in the 
superdomain of $D$ have $v$ as value for $m_{n}^{f(D)}(D)$, i.e.,

$$
\operatorname{Dfatherknows}(D, v)=\left\langle\forall n: n \in \operatorname{Dnodes}(f(D)):: m_{n}^{f(D)}(D)=v\right\rangle
$$

- Let $n \in \operatorname{Dnodes}(D)$. Node $n$ has finished for $D$ with value $v$ if and only if the value $v$, as stored by $n$ for $D$, equals the maximum of the values of the subdomains as kept by $n$.

$$
\begin{aligned}
& \text { Nfinished }(n, D, v)= \\
& \begin{cases}v=m_{n}^{D}(D)=\operatorname{inp}(D) & \text { if Dnodes }(D)=\{D\} \\
v=m_{n}^{D}(D)=\left\langle\max C: C \triangleleft D:: m_{n}^{D}(C)\right\rangle & \text { otherwise }\end{cases}
\end{aligned}
$$

- The algorithm on a division tree has finished for a tree-node if and only if its value equals the maximum of the values of its terminated sons (as stored by the tree-node). In this case, the (distributed) algorithm has finished for a domain if and only if all nodes contained in the domain have finished for the domain and all subdomains have terminated (which is defined next). Dhfinished $(D, v)$ holds if and only if the algorithm has finished for $D$ with value $v$, i.e.,

$$
\begin{aligned}
\operatorname{Dhfinished}(D, v)^{*}= & (\langle\forall n: n \in \operatorname{Dnodes}(D):: N \text { Ninished }(n, D, v)\rangle \wedge \\
& \left.\left\langle\forall C: C \triangleleft D::\left\langle\exists v_{c}:: \text { Dhterm }\left(C, v_{c}\right)\right\rangle\right\rangle\right)
\end{aligned}
$$

- The algorithm on a division tree has terminated for a tree-node if and only if the algorithm has finished for the tree-node and the value of the tree-node is known by the father. Similarly, the distributed algorithm has terminated for a domain if and only if the algorithm has finished for the domain and the value of the domain (which all nodes contained in the domain have) is known by all nodes contained in the superdomain. $\operatorname{Dhterm}(D, v)$ holds if and only if the algorithm has terminated for domain $D$ with value $v$, i.e.,

$$
\operatorname{Dhterm}(D, v)=\operatorname{Dhfinished}(D, v) \wedge D \text { fatherknows }(D, v)
$$

- equal $(D, S)$ holds if and only if all nodes contained in the superdomain of $D$ have the same value $S$ as sequence number for $D$, i.e.,

$$
\begin{aligned}
& \text { equal }(D, S)=\left\langle\forall n: n \in \text { Dnodes }(f(D)):: S_{n}(D)=S\right\rangle \\
& \operatorname{maxs}(D, S)=\left(S=\left\langle\max n: n \in \operatorname{Dnodes}(f(D)):: S_{n}(D)\right\rangle\right)
\end{aligned}
$$

\subsubsection{The Solution Strategy: Formal Description}

A formal specification of an algorithm on a hierarchically divided network is derived. The specification follows from the informal description of the algorithm and the formal specification of the algorithm on a division tree.

When every domain is capable of performing the actions the corresponding tree-node in the algorithm on a division tree does, every domain will keep the maximum of the inputs contained in the domain. In other words, the actions performed by the domains must satisfy the conditions SP3, SP4, SP5 and SP6. However, domains are not able to perform any actions. As described in Section 6.1, the actions of a domain are simulated by the (physical) nodes contained in the domain. These observations result in the following conditions. 
If the algorithm has finished for domain $D$ (Dhfinished $(D, v)$ holds), no node contained in $D$ may change its computed maximum of $D$ or of domains in $D$ unless an input contained in $D$ has changed.

$(\operatorname{Dhfinished}(D, v) \wedge$ Dnodesval $(D)=\vec{M})$ unless $($ Dnodesval $(D) \neq \vec{M})$

If the algorithm has terminated in domain $D$, no node contained in $f(D)$ may change its computed maximum of $D$ as long as the algorithm has finished for $D$.

$\operatorname{Dhterm}(D, v)$ unless $\neg$ Dhfinished $(D, v)$

When the algorithm has terminated in every subdomain of $D$, all nodes contained in $D$ are able to compute the maximum input contained in $D$. After the computations the algorithm may have finished for $D$. However, if during the computations at least one input contained in $D$ changed, the algorithm has not yet finished for a subdomain of $D$.

$$
\begin{aligned}
\left\langle\forall C: C \triangleleft D:: \text { Dhterm }\left(C, v_{c}\right)\right\rangle & \mapsto \\
(\langle\exists v:: \text { Dhfinished }(D, v)\rangle & \left.\vee\left\langle\exists C: C \triangleleft D:: \neg \text { Dhfinished }\left(C, v_{c}\right)\right\rangle\right)
\end{aligned}
$$

The last progress property, as given in Section 5.2, states that if the algorithm has finished for tree-node $D$, within finite time the algorithm must terminate for $D$ or the algorithm is not finished for $D$ (because of a change in an input). This property must also hold for domains in a hierarchically divided network.

$$
\text { Dhfinished }(D, v) \mapsto(\operatorname{Dhterm}(D, v) \vee \neg D h f i n i s h e d(D, v))
$$

The specification of obtaining and maintaining the maximum value of the inputs at the nodes in a domain $D$ by all nodes in $D$, can be formally formulated as follows:

SP10: $($ Dhfinished $(D, v) \wedge$ Dnodesval $(D)=\vec{M})$ unless $($ Dnodesval $(D) \neq \vec{M})$

SP11: Dhterm $(D, v)$ unless $\neg$ Dhfinished $(D, v)$

SP12: $\left\langle\forall C: C \triangleleft D\right.$ :: Dhterm $\left.\left(C, v_{c}\right)\right\rangle \mapsto$

$\left(\langle\exists v::\right.$ Dhfinished $(D, v)\rangle \vee\left\langle\exists C: C \triangleleft D:: \neg\right.$ Dhfinished $\left.\left.\left(C, v_{c}\right)\right\rangle\right)$

SP13: Dhfinished $(D, v) \mapsto(\operatorname{Dhterm}(D, v) \vee \neg D$ Dhfinished $(D, v))$

\subsubsection{Proof of Correctness of the Solution Strategy}

To prove the correctness of the solution strategy, it suffices to prove that the relations SP3, SP4, SP5 and SP6 are met by any strategy that satisfies conditions SP10, SP11, SP12 and SP13. As stated before, every tree-node corresponds to a domain in a hierarchically divided network. A tree-node has a value for some variable if and only if the corresponding domain has the value for the variable, i.e., all nodes contained in the domain have the value for the variable. From this observation, SP3, SP4, SP5 and SP6 directly follow from SP10, SP11, SP12 and SP13, respectively.

\subsubsection{The Solution Strategy: Formal Description Refined}

The given solution strategy is not yet in a form that can be directly translated to a (distributed) program. In order to come up with such a solution strategy the properties SP10 through SP13 must be refined. 
First, the properties relating Dhfinished, i.e., SP10 and SP12, are refined. To satisfy SP10, by some node $n$, two cases are distinguished. If node $n$ has finished its computation for $n$ itself, i.e., $m_{n}^{n}(n)=\operatorname{inp}(n)$, the value of $m_{n}^{n}(n)$ should not change unless the input of $n$ has changed.

$$
[(n=D) \wedge\langle\exists v \quad:: \text { Nfinished }(n, D, v)\rangle] \text { unless }(\operatorname{inp}(D) \neq v)
$$

The second case concerns the computation for some domain $D$ by node $n$, with $n \in \operatorname{Dnodes}(D) \wedge n \neq D$. When the algorithm has terminated for every subdomain of $D$ (thus $n$ is aware of the maximum input contained in each subdomain of $D$ ), and $n$ has computed the maximum input contained in $D, n$ should not change this computed maximum for $D$ unless the algorithm is not finished anymore for a subdomain of $D$ (because of a change in an input contained in a subdomain of D), i.e.,

$\left[(n \in\right.$ Dnodes $(D)) \wedge(n \neq D) \wedge\left\langle\forall C \quad: C \triangleleft D \quad:: \operatorname{Dhterm}\left(C, v_{C}\right)\right\rangle \wedge\langle\exists v \quad::$ Nfinished $(n, D, v)\rangle]$ unless $\left\langle\exists C: C \triangleleft D:: \neg\right.$ Dhfinished $\left.\left(C, v_{C}\right)\right\rangle$

Relation SP12 states that if the algorithm has terminated for every subdomain of $D$, within finite time the algorithm will finish for $D$ (i.e., every node contained in $D$ has computed the maximum of the maximum inputs contained in the subdomains), or the algorithm has not finished in a subdomain of $D$ (because of a change in an input contained in $D$ ). By ensuring that a node is always finished in finite time for every domain it is contained in, SP12 is satisfied. This results in the following relation:

$$
(n \in \text { Dnodes }(D)) \text { ensures }\langle\exists v \quad:: \text { Nfinished }(n, D, v)\rangle
$$

Next, the properties SP11 and SP13, relating Dhterm, are refined. The algorithm has terminated for domain $D$, if and only if the algorithm has finished for $D$ and all nodes contained in $f(D)$ are aware of the maximum input contained in $D$. In other words, the algorithm has terminated for $D$, if and only if the algorithm has finished for $D$ and all nodes $n \in \operatorname{Dnodes}(f(D))$ have set $m_{n}^{f(D)}(D)$ to the maximum input contained in $D$. After the algorithm has finished for $D$, for all nodes $n \in$ Dnodes $(D), m_{n}^{D}(D)$ equals the maximum input contained in $D$. Thus, after the algorithm has finished for $D$, every node $n \in D$ nodes $(D)$ can set $m_{n}^{f(D)}(D)$ to the maximum input contained in $D$ by assigning $m_{n}^{D}(D)$ to $m_{n}^{f(D)}(D)$. However, as described in Section 6.1.2, to inform the nodes contained in $f(D) \backslash D$ about the maximum input contained in $D$, is more complicated. For that purpose, sequence numbers are introduced.

The higher the sequence number, the more recent is the corresponding information. Therefore, every sequence number, kept by some node, must be nondecreasing, i.e.,

$$
\left(S_{n}(D)=S\right) \text { unless }\left(S_{n}(D)>S\right)
$$

Consider a node $n \in$ Dnodes $(f(D) \backslash D)$. Suppose $n$ obtained its most recent information about $D$ from neighbour node $n^{\prime} \in \operatorname{Dnodes}(f(D))$. At that moment, $\left(\left(S_{n}(D)=S_{n^{\prime}}(D)\right) \wedge\left(m_{n}^{f(D)}(D)=m_{n^{\prime}}^{f(D)}(D)\right)\right)$. However, $n^{\prime}$ might have increased $S_{n^{\prime}}(D)$ as a result of one of the following scenarios:

- $n^{\prime} \in \operatorname{Dnodes}(D)$ and $n^{\prime}$ computed a new value for $D$. In this case, $n^{\prime}$ has information that is more recent. Thus, $m_{n^{\prime}}^{f(D)}(D)$ must be set to this value and $S_{n^{\prime}}(D)$ must be increased, resulting in $\left(S_{n^{\prime}}(D)>S_{n}(D)\right)$. 
- $n^{\prime} \notin D$ Dodes $(D)$ and $n^{\prime}$ obtained information from a neighbour $n^{\prime \prime}(\neq n)$ that is more recent, i.e., $S_{n^{\prime}}(D)<S_{n^{\prime \prime}}(D)$. In this case, $S_{n^{\prime}}(D)$ and $m_{n^{\prime}}^{f(D)}(D)$ must be set to $S_{n^{\prime \prime}}(D)$ and $m_{n^{\prime \prime}}^{f(D)}(D)$, respectively. Consequently, also in this case $\left(S_{n^{\prime}}(D)>S_{n}(D)\right)$.

In other words, there is always a neighbour node $n^{\prime} \in \operatorname{Dnodes}(f(D))$ of $n$ such that $\left[\left(S_{n}(D)=S_{n^{\prime}}(D) \wedge m_{n}^{f(D)}(D)=m_{n^{\prime}}^{f(D)}(D)\right) \vee\left(S_{n}(D)<S_{n^{\prime}}(D)\right)\right]$. This same property holds for $n^{\prime}$ (with neighbour $n^{\prime \prime}$ ) if $n^{\prime} \notin$ Dnodes $(D)$. This suggests the following invariant,

$$
\langle\forall n: n \in \operatorname{Dnodes}(f(D) \backslash D):: \operatorname{chain}(n, D)\rangle
$$

with

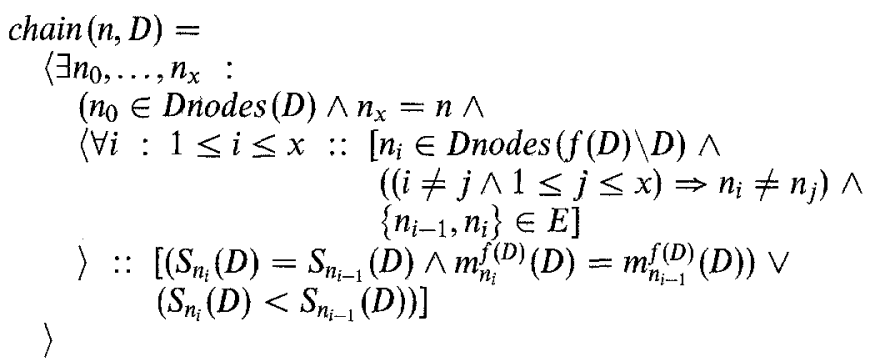

If the algorithm has finished for $D$ and some node $n$ is aware of the newest information about $D$, i.e., the maximum input and/or the maximum sequence number contained in $D$, it should not change this information until an input has changed in $D$, in which case the algorithm has not finished anymore for $D$. This results in the following properties:

$\left[(n \in \operatorname{Dnodes}(D)) \wedge\right.$ Dhfinished $\left.(D, v) \wedge\left(m_{n}^{f(D)}(D)=m_{n}^{D}(D)\right)\right]$ unless $\neg$ Dhfinished $(D, v)$

and

$\left[(n \in \operatorname{Dnodes}(f(D))) \wedge \operatorname{Dhfinished}(D, v) \wedge \operatorname{maxs}\left(D, S_{n}(D)\right)\right]$ unless $\neg$ Dhfinished $(D, v)$

Next, the progress properties are given. If some node $n \in \operatorname{Dnodes}(D)$ has changed the value for $D$, i.e., $m_{n}^{D}(D)$ has changed, within finite time $m_{n}^{f(D)}(D)$ must be set to $m_{n}^{D}(D)$. Thus,

$$
(n \in \text { Dnodes }(D)) \text { ensures }\left(m_{n}^{f(D)}(D)=m_{n}^{D}(D)\right)
$$

If a neighbour node of $n \in \operatorname{Dnodes}(f(D))$ has a higher sequence number for $D$, within finite time $n$ must have updated its sequence number to a value at least as high,

$$
\left(\left\{n, n^{\prime}\right\} \in E \wedge n, n^{\prime} \in \operatorname{Dnodes}(f(D))\right) \text { ensures }\left(S_{n}(D) \geq S_{n^{\prime}}(D)\right)
$$

The complete specification consists of the following properties:

SP14: $[(n=D) \wedge\langle\exists v::$ Nfinished $(n, D, v)\rangle]$ unless $(\operatorname{inp}(D) \neq v)$

SP15: $\left[(n \in\right.$ Dnodes $(D)) \wedge(n \neq D) \wedge\left\langle\forall C: C \triangleleft D \quad::\right.$ Dhterm $\left.\left(C, v_{C}\right)\right\rangle \wedge\langle\exists v \quad::$

$N$ finished $(n, D, v)\rangle]$ unless $\left\langle\exists C: C \triangleleft D:: \neg\right.$ Dhfinished $\left.\left(C, v_{C}\right)\right\rangle$

SP16: $\left[(n \in \operatorname{Dnodes}(D)) \wedge \operatorname{Dhfinished}(D, v) \wedge\left(m_{n}^{f(D)}(D)=m_{n}^{D}(D)\right)\right]$ unless $\neg$ Dhfinished $(D, v)$ 
SP17: $\left[(n \in\right.$ Dnodes $\left.(f(D))) \wedge \operatorname{Dhfinished}(D, v) \wedge \operatorname{maxs}\left(D, S_{n}(D)\right)\right]$ unless $\neg$ Dhfinished $(D, v)$

SP18: $(n \in \operatorname{Dnodes}(D))$ ensures $\langle\exists v \quad::$ Nfinished $(n, D, v)\rangle$

SP19: $(n \in$ Dnodes $(D))$ ensures $\left(m_{n}^{f(D)}(D)=m_{n}^{D}(D)\right)$

SP20: $\left(S_{n}(D)=S\right)$ unless $\left(S_{n}(D)>S\right)$

SP21: $\left(\left\{n, n^{\prime}\right\} \in E \wedge n, n^{\prime} \in \operatorname{Dnodes}(f(D))\right)$ ensures $\left(S_{n}(D) \geq S_{n^{\prime}}(D)\right)$

SP22: Invariant

$\langle\forall n: n \in$ Dnodes $(f(D) \backslash D)::$ chain $(n, D)\rangle$

\subsubsection{Proof of Correctness of the Solution Strategy}

To prove the correctness of the solution strategy it is sufficient to prove that the relations SP10, SP11, SP12 and SP13 are met by any strategy that satisfies conditions SP14 through SP22. In Appendix C the formal proof is given, here, we give an outline of the proof.

First, it is proven that SP10 is met. SP15 states that every node $n$ contained in $D$, stops its computation for $D$ if the algorithm has terminated for every subdomain of $D$ and $m_{n}^{D}(D)$ equals the maximum input contained in $D$, until the algorithm is no more finished for a subdomain of $D$. The algorithm has finished for a domain $D$ if and only if the algorithm has terminated for every subdomain of $D$ and for every node $n$ contained in $D, m_{n}^{D}(D)$ equals the maximum input. From the above observations, it directly follows that if the algorithm has finished for $D$, which is not a node, it remains finished for $D$, until the algorithm is no more finished for a subdomain of $D$. With a simple induction argument, it can now be proven that if the algorithm has finished for $D$, it remains finished for $D$, until an input contained in $D$ has changed. (The base case follows directly from SP14). This proves SP10.

SP16 states that once the algorithm has finished for $D$ with value $v$ and for a node $n$ contained in $D, m_{n}^{f(D)}(D)=m_{n}^{D}(D)=v, n$ does not change its value for $m_{n}^{f(D)}(D)$ until the algorithm is not finished anymore for $D$. SP17 states that once the algorithm has finished for $D$ and for a node $n$ contained in $f(D), S_{n}(D)$ equals the maximum sequence number for $D$ contained in $f(D)$, then $n$ does not change $S_{n}(D)$ until the algorithm is not finished for $D$ anymore. Thus, from SP16 and SP17, it follows that if algorithm has finished for $D$ with value $v$ and all nodes $n$ contained in $D$ have $m_{n}^{f(D)}(D)=v$ and all nodes contained in $f(D)$ have the same sequence number for $D$, nothing changes until the algorithm is not finished for $D$ anymore. From this observation and invariant SP22, SP11 follows.

SP18 states that every node contained in $D$ finishes its computation for $D$ eventually. As is proven before, the algorithm has terminated for a domain until the algorithm is not finished for the domain anymore. Thus, if the algorithm has terminated for every subdomain of a domain $D$, within finite time: the algorithm has terminated for every subdomain of $D$ and every node contained in $D$ has finished its computation for $D$, or the algorithm is not finished anymore for a subdomain of $D$. From the definition of finished, SP12 follows.

From invariant $\mathrm{SP} 22$, the algorithm has terminated for some domain $D$, if the following three properties hold: The algorithm has finished for $D$, for all nodes $n$ contained in $D, m_{n}^{f(D)}(D)=m_{n}^{D}(D)$ and equal $(D, S)$. Therefore, to prove SP13, first the following property is proven: If the algorithm has finished for $D$, within finite time: The algorithm has finished for $D$ and for all nodes $n$ contained in $D$, $m_{n}^{f(D)}(D)=m_{n}^{D}(D)$, or the algorithm is not finished for $D$ anymore. This property 
follows from SP20, which states that for every node $n$ contained in $D$, within finite time, $m_{n}^{f(D)}(D)=m_{n}^{D}(D)$.

Next, some properties of the sequence numbers are proven. The first property is that at any moment, where $\max (D, S)$ holds (i.e., $S$ is the maximum sequence number for $D$ ), within finite time all nodes contained in $f(D)$ have $S$ for the sequence number for $D$ (equal $(D, S)$ holds), or the maximum sequence number for $D$ has increased. This property has been proven using SP21, which states that always within finite time the sequence number for $D$ at some node $n$ contained in $f(D)$ is at least as big as the sequence number for $D$ at every neighbour node contained in $f(D)$.

SP17 states that if the algorithm has finished for $D$, the maximum sequence number for $D$ does not increase, until the algorithm is not finished for $D$ anymore. From SP17 and the property proven above, the following holds: if the algorithm has finished for $D$, within finite time the algorithm has finished for $D$ and equal $(D, S)$ holds, or the algorithm is not finished for $D$ anymore.

By combining the above results, SP13 follows.

\subsubsection{Derivation of a Program from the Specification}

We will give a program, where each statement implements a progress condition. Each statement must also preserve the invariant and the unless relations.

Statements derived from SP18:

$$
\begin{array}{rlrl}
\langle(\square n, D: & n \in D \text { nodes }(D):: & \\
& m_{n}^{D}(D):= & & \\
& \operatorname{inp}(D) & & \text { if } n=D \sim \\
& \left.\max C: C \triangleleft D:: m_{n}^{D}(C)\right\rangle & & \text { if }(n \neq D)
\end{array}
$$

Statements derived from SP19:

$\left\langle\square n, D: n \in\right.$ Dnodes $\left.(D) \quad:: m_{n}^{f(D)}(D):=m_{n}^{D}(D)\right\rangle$

However, if $n$ is a border node of $D$, invariant SP22 is violated after the execution of the statement. Therefore, to preserve invariant SP22, after border node $n$ changed $m_{n}^{f(D)}(D), S_{n}(D)$ must be increased, i.e.,

$$
\begin{array}{rlrl}
\langle(\square n, D: n \in \text { Dnodes }(D):: & \\
m_{n}^{f(D)}(D), S_{n}(D):= & m_{n}^{D}(D), S_{n}(D)+1 & \text { if }\left(\left(m_{n}^{f(D)} \neq m_{n}^{D}(D)\right) \wedge\right. \\
& & (n \in \operatorname{Border}(D))) \sim \\
& m_{n}^{D}(D), S_{n}(D) & \text { if }\left(\left(m_{n}^{f(D)} \neq m_{n}^{D}(D)\right) \wedge\right. \\
& & (n \notin \operatorname{Border}(D)))
\end{array}
$$

Statements derived from SP21:

$$
\begin{aligned}
\left\langle\left[ n, n^{\prime}, D\right.\right. & : n, n^{\prime} \in \text { Dnodes }(f(D)) \wedge\left\{n, n^{\prime}\right\} \in E \quad:: S_{n}(D):=S_{n^{\prime}}(D) \\
& \text { if }\left(S_{n^{\prime}}(D)>S_{n}(D)\right)
\end{aligned}
$$

However, if $n$ is contained in $f(D) \backslash D$, invariant SP22 can be violated. Therefore, to preserve invariant SP22, node $n$ must set $m_{n}^{f(D)}(D)$ to $m_{n^{\prime}}^{f(D)}(D)$ if $S_{n}(D)$ is set to $S_{n^{\prime}}(D)$, i.e., 


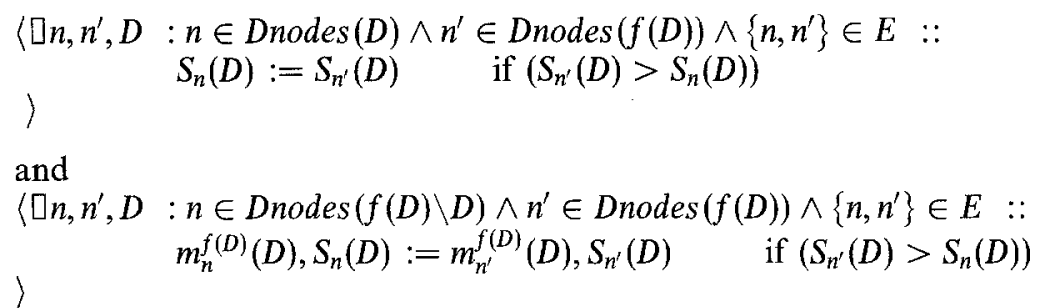

To show that these statements do not violate the safety properties is rather straightforward and left to the reader.

The complete program follows.

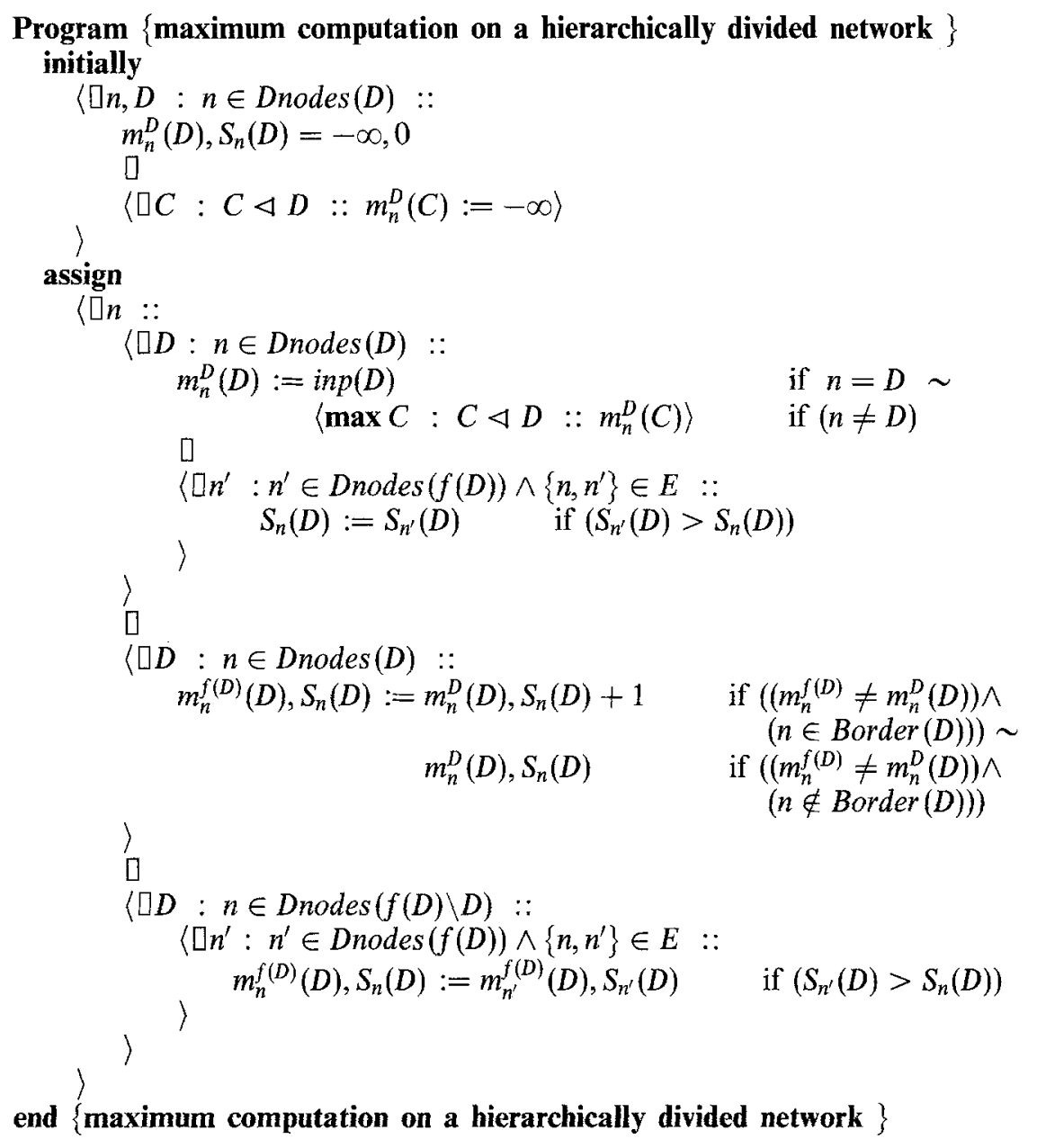

Similarly, as in Section 5.3, this program can be refined such that it is equivalent to the informally described algorithm in Section 6.1.2. The program can be implemented on a distributed system in the obvious way. The variables $m_{n}^{D}(D), m_{n}^{f(D)}(D)$ and $S_{n}(D)$ are then local to $n$. A change in one of these variables 
is made known to the neighbours of $n$ contained in the superdomain of $D$, by sending it over the channels to these neighbours. Moreover, by adding boolean variables the program can be refined to simulate the execution of the informally described algorithm.

\section{Conclusion}

In this paper a distributed algorithm on a hierarchically divided network is derived. Every node in the network has an associated input value and a variable in which it keeps the maximum of all inputs. These inputs may change by external causes. Within finite time after the last change, every node has updated its local copy of the maximum value of the inputs.

The algorithm is developed in stages. After the problem description, an abstract algorithm on a (virtual) division tree is given. Next, the algorithm is transformed in the distributed algorithm that operates on a hierarchically divided network.

The stages are described informally as well as formally, using the UNITYmethod. Each algorithm is first described informally. It is our experience that the informal description of the resulting algorithm may be very helpful in the design and study of the algorithm using UNITY.

It is our hope, however, that new calculi to be developed may be used to combine the presentation of the insights and their formal encoding. As such this problem is also posed as a challenge to calculi designers.

We have used the techniques in this article to design several distributed algorithms on hierarchically divided networks. Our goal is to design an inherently safe packet switching network. This network will be hierarchically divided upon which the distributed hierarchical routing algorithm operates, which was introduced in [LUS89]. Before designing and verifying an algorithm with which the nodes in the network recompute their routing information after a topology change has occurred, we decided to first gain some experience on a simpler problem.

The problem of maintaining the maximum value of a bag of inputs and the problem of keeping topological information about a hierarchically divided network bear some similarities. The network can be considered as a set of nodes. With each node in this set corresponds a possibly changing set of paths from a source node to that node. Every node must, for every other destination node in the set, obtain and maintain the optimal path.

The problem of maintaining this routing information is for two reasons more complex than the problem of keeping the maximum value. In the first place, it deals with a set of sets, viz., for each node in the set of destination nodes there is a set of paths to it.

The second reason is that every node has to deal with a different set of paths for the same destination node. In keeping the maximum value of a bag of inputs, all nodes have to deal with the same bag of integers.

We are, however, convinced that the (formal) derivation of a distributed algorithm for keeping the maximum value of a frequently changing bag of inputs, that operates on a hierarchically divided network, will help in the (formal) derivation of an algorithm with which nodes in a hierarchically divided network keep their routing information. 


\section{Acknowledgement}

The authors thank Arjen Uittenbogaard, Kaisa Sere and Gerard Tel for their help during the course of this research.

\section{References}

[ChM88] Chandy, K.M. and Misra, J.: Parallel Program Design - A Foundation. Addison-Wesley Publishing Company, Inc., 1988.

[Kna90] Knapp, E.: An exercise in the formal derivation of parallel programs: Maximum flows in graphs. ACM Transactions on Programming Languages and Systems, 12(2):203-223, April 1990.

[LUS89] Lentfert, P.J.A. Uittenbogaard, A.H. Swierstra, S.D. and Tel, G.: Distributed hierarchical routing. Technical Report RUU-CS-89-5, Utrecht University, March 1989. Also in: P.M.G. Apers et al. (Eds.). Proceedings CSN89. Utrecht, November 9-10, 1989.

[Mis89] Misra, J.: Specifications of concurrently accessed data. In J. L. A. van de Snepscheut, editor, Proceedings of the Conference on the Mathematics of Program Construction, Groningen, pages 91-114, New York, June 1989. Springer-Verlag.

[Per83] Perlman, R. Fault-tolerant broadcast of routing information. Computer Networks, 7:395-405, December 1983.

[San91] Sanders, B.A. Eliminating the substitution axiom from UNITY logic. Formal aspects of Computing, 3(2) :189-205, 1991.

[Sta88] Staskauskas, M.G. The formal specification and design of a distributed electronic funds transfer system. IEEE Trans. Comp., 37(12):1515-1528, December 1988.

\section{A. Theorems about UNITY Operators}

In this section some known results about the UNITY operators are presented, which are used in the following appendices. The results are given without proof. For detailed proofs, the interested reader is referred to [ChM88]. Note that in the proofs the standard definitions of the UNITY operators are used. However, these proofs can simply be adapted to the definitions used in this article.

In the following, $p, q, r$ and $b$ denote predicates.

Antireflexivity of unless:

$p$ unless $\neg p$

Simple Conjunction:

$\left((p\right.$ unless $q) \wedge\left(p^{\prime}\right.$ unless $\left.\left.q^{\prime}\right)\right) \Rightarrow\left(\left(p \wedge p^{\prime}\right)\right.$ unless $\left.\left(q \vee q^{\prime}\right)\right)$

Conjunction:

$\left((p\right.$ unless $q) \wedge\left(p^{\prime}\right.$ unless $\left.\left.q^{\prime}\right)\right) \Rightarrow\left[\left(p \wedge p^{\prime}\right)\right.$ unless $\left.\left(\left(p \wedge q^{\prime}\right) \vee\left(p^{\prime} \wedge q\right) \vee\left(q \wedge q^{\prime}\right)\right)\right]$

Implication theorem:

$$
(p \Rightarrow q) \Rightarrow(p \mapsto q)
$$

Weakening the right side:

$$
(p \mapsto(q \wedge r)) \Rightarrow(p \mapsto r)
$$

PSP (Progress-Safety-Progress) theorem:

$$
((p \mapsto q) \wedge(r \text { unless } b)) \Rightarrow((p \wedge r) \mapsto((q \wedge r) \vee b))
$$


Cancellation theorem:

$((p \mapsto q \vee b) \wedge(b \mapsto r)) \Rightarrow(p \mapsto q \vee r)$

Completion theorem:

For any finite set of predicates $p_{i}, q_{i}$, where $0 \leq i<N$ :

$\left(\left\langle\forall i:: p_{i} \mapsto q_{i} \vee b\right\rangle \wedge\left\langle\forall i:: q_{i}\right.\right.$ unless $\left.\left.b\right\rangle\right) \Rightarrow\left[\left\langle\forall i \quad:: p_{i}\right\rangle \mapsto\left(\left\langle\forall i:: q_{i}\right\rangle \vee b\right)\right]$

Generalization of the Completion theorem:

For any finite set of predicates $p_{i}, q_{i}, r_{i}, b_{i}$, where $0 \leq i<N$ :

$\left(\left\langle\forall i:: p_{i} \mapsto q_{i} \vee r_{i}\right\rangle \wedge\left\langle\forall i:: q_{i}\right.\right.$ unless $\left.\left.b_{i}\right\rangle\right) \Rightarrow$

$\left\langle\forall i:: p_{i}\right\rangle \mapsto\left(\left\langle\forall i:: q_{i}\right\rangle \vee\left\langle\exists i:: r_{i} \vee b_{i}\right\rangle\right)$

In [San91], substitution rules are given that can replace the substitution axiom (as given in [ChM88]). Below, all occurrences of Prop can be replaced by unless, ensures or $\mapsto$. Furthermore, $z$ and $y$ denote either both variables or predicates, and $P$ and $Q$ map $z$ to predicates. Then, for a single program, the following theorem holds:

Substitution:

$[(P . z$ Prop $Q . z)] \wedge$ (invariant $r) \wedge(r \Rightarrow(z=y))] \Rightarrow(P . y$ Prop $Q . y)$

\section{B. Formal Proof of Solution Strategy for Division Tree}

It is proven that any strategy satisfying conditions SP3, SP4, SP5 and SP6 (Section 5.2.2) also meet the relations SP1 and SP2 (Section 4.1.2). In the proof a number of known results are used, which are given in Appendix A.

Lemma B.1. hterm $(D, v)=\left[\left(v=m^{D}(D)=\langle\max n: n \in \operatorname{leaves}(D):: \operatorname{inp}(n)\rangle\right) \wedge\right.$ fatherknows $(D, v) \wedge\left\langle\forall C: C \triangleleft D::\right.$ hterm $\left.\left.\left(C, m^{C}(C)\right)\right\rangle\right]$.

Proof. We use induction on the structure of the division-tree.

Base: $D$ is a leaf. From the definition of $\operatorname{hterm}(D, v)$, with $D$ a leaf of the division-tree, this case directly follows.

Step: Suppose $D$ is not a leaf and the lemma holds for all sons of $D$, i.e., $\left\langle\forall C: C \triangleleft D::\right.$ hterm $\left(C, v_{c}\right)=\left[\left(v_{c}=m^{C}(C)=\langle\max n: n \in\right.\right.$ leaves $(C) \quad::$ inp $(n)\rangle) \wedge$ fatherknows $\left(C, v_{c}\right) \wedge\left\langle\forall B: B \triangleleft C:\right.$ hterm $\left.\left.\left.\left(B, m^{B}(B)\right)\right\rangle\right]\right\rangle$.

Then the following holds:

$$
\begin{aligned}
& \text { hterm }(D, v) \\
&= {[\text { hfinished }(D, v) \wedge \text { fatherknows }(D, v)] } \\
&= {\left[\left(v=m^{D}(D)=\left\langle\max C: C \triangleleft D:: m^{D}(C)\right\rangle \wedge\right.\right.} \\
&\left.\left\langle\forall C: C \triangleleft D:: \text { hterm }\left(C, m^{C}(C)\right)\right\rangle \wedge \text { fatherknows }(D, v)\right] \\
&\left\{\text { hterm }\left(C, m^{C}(C)\right) \Rightarrow\left(m^{D}(C)=m^{C}(C)\right)\right\} \\
&=\left[\left(v=m^{D}(D)=\left\langle\max C: C \triangleleft D:: m^{C}(C)\right\rangle\right) \wedge\right. \\
&\left.\quad\left\langle\forall C: C \triangleleft D:: \text { hterm }\left(C, m^{C}(C)\right)\right\rangle \wedge \text { fatherknows }(D, v)\right] \\
&\{\text { Induction Hypothesis }\} \\
&=\left[\left(v=m^{D}(D)\right.\right. \\
&\quad=\langle\max C: C \triangleleft D::\langle\max n: n \in \text { leaves }(C):: \text { inp }(n)\rangle\rangle) \wedge \\
&\left.\quad\left\langle\forall C: C \triangleleft D:: \text { hterm }\left(C, m^{C}(C)\right)\right\rangle \wedge \text { fatherknows }(D, v)\right]
\end{aligned}
$$




$$
\begin{aligned}
= & {\left[\left(v=m^{D}(D)=\langle\max n: n \in \text { leaves }(D):: \operatorname{inp}(n)\rangle\right) \wedge\right.} \\
& \left.\left\langle\forall C: C \triangleleft D:: \text { hterm }\left(C, m^{C}(C)\right)\right\rangle \wedge \text { fatherknows }(D, v)\right] .
\end{aligned}
$$

This proves the induction step, thereby proving the lemma.

Corollary B.1. If $R$ is the root of the division tree corresponding to a hierarchical division on $G$, it is possible to define: $\operatorname{hterm}(R, v)=\operatorname{term}(G, v)$.

In the following we will use this definition. SP1 directly follows from this and SP3. We will now prove that SP2 is met.

\section{Lemma B.2.}

$$
\begin{gathered}
\left\langle\forall C: C \triangleleft D::\left(\text { hinished }\left(C, v_{c}\right) \wedge\left(\text { leavesval }(C)=\vec{M}_{c}\right)\right)\right\rangle \mapsto \\
\left(\left\langle\forall C: C \triangleleft D:: \text { hterm }\left(C, v_{c}\right)\right\rangle\right. \\
\left.\vee \neg\left\langle\forall C: C \triangleleft D:: \text { leavesval }(C)=\vec{M}_{c}\right\rangle\right) .
\end{gathered}
$$

Proof.

PSP theorem on SP3 and SP6:

(hfinished $\left(C, v_{c}\right) \wedge\left(\right.$ leavesval $\left.\left.(C)=\vec{M}_{c}\right)\right) \mapsto$

$\left(\left[\left(\right.\right.\right.$ hterm $\left(C, v_{c}\right) \vee \neg$ hinished $\left.\left(C, v_{c}\right)\right) \wedge \operatorname{hinished}\left(C, v_{c}\right) \wedge$

$$
\begin{gathered}
\left.\left(\text { leavesval }(C)=\vec{M}_{c}\right)\right] \\
\left.\vee \neg\left(\text { leavesval }(C)=\vec{M}_{c}\right)\right)
\end{gathered}
$$

Rewriting the right side:

$$
\begin{array}{r}
\left(\text { hfinished }\left(C, v_{c}\right) \wedge\left(\text { leavesval }(C)=\vec{M}_{c}\right)\right) \mapsto \\
\left(\text { hterm }\left(C, v_{c}\right) \vee \neg\left(\text { leavesval }(C)=\vec{M}_{\mathcal{c}}\right)\right)
\end{array}
$$

Conjunction over the $C^{\prime}$ s on the above:

$$
\begin{aligned}
\langle\forall C: C \triangleleft D:: & \left(\text { hfinished }\left(C, v_{c}\right) \wedge\left(\text { leavesval }(C)=\vec{M}_{c}\right)\right) \mapsto \\
\left(\text { hterm }\left(C, v_{c}\right) \vee \neg(\text { leavesval }(C)\right. & \left.\left.=\vec{M}_{c}\right)\right)
\end{aligned}
$$

\section{From SP4:}

$\left\langle\forall C: C \triangleleft D::\right.$ hterm $\left(C, v_{c}\right)$ unless $\neg\left(\right.$ hfinished $\left.\left.\left(C, v_{c}\right)\right)\right\rangle$

The Generalization of the Completion theorem on the above two:

$$
\begin{aligned}
\left\langle\forall C: C \triangleleft D:: \text { hfinished }\left(C, v_{c}\right) \wedge\left(\text { leavesval }(C)=\vec{M}_{c}\right)\right\rangle \mapsto \\
\quad\left(\left\langle\forall C: C \triangleleft D:: \text { hterm }\left(C, v_{c}\right)\right\rangle\right. \\
\left.\quad \vee \neg\left\langle\forall C: C \triangleleft D::\left(\text { leavesval }(C)=\vec{M}_{c}\right) \wedge \text { hfinished }\left(C, v_{c}\right)\right\rangle\right)
\end{aligned}
$$

From SP3, using Simple Conjunction:

$\left\langle\forall C: C \triangleleft D::\right.$ hfinished $\left(C, v_{c}\right) \wedge\left(\right.$ leavesval $\left.\left.(C)=\vec{M}_{c}\right)\right\rangle$ unless

$$
\neg\left\langle\forall C: C \triangleleft D:: \text { leavesval }(C)=\vec{M}_{c}\right\rangle
$$

The PSP theorem on the above two:

$$
\begin{aligned}
& \left\langle\forall C: C \triangleleft D:: \text { hfinished }\left(C, v_{c}\right) \wedge\left(\text { leavesval }(C)=\vec{M}_{c}\right)\right\rangle \mapsto \\
& {\left[\left(\left(\left\langle\forall C: C \triangleleft D:: \text { hterm }\left(C, v_{c}\right)\right\rangle \vee\right.\right.\right.} \\
& \left.\left.\neg\left\langle\forall C: C \triangleleft D:: \text { (leavesval }(C)=\vec{M}_{c}\right) \wedge \text { hfinished }\left(C, v_{c}\right)\right\rangle\right) \wedge \\
& \left.\left.\left\langle\forall C: C \triangleleft D:: \text { (leavesval }(C)=\vec{M}_{c}\right) \wedge \text { hfinished }\left(C, v_{c}\right)\right\rangle\right)
\end{aligned}
$$




$$
\left.\vee \neg\left\langle\forall C: C \triangleleft D:: \text { leavesval }(C)=\vec{M}_{c}\right\rangle\right]
$$

Rewriting the right side:

$\left\langle\forall C: C \triangleleft D::\right.$ hfinished $\left(C, v_{c}\right) \wedge$ (leavesval $\left.\left.(C)=\vec{M}_{c}\right)\right\rangle \mapsto$

$$
\begin{gathered}
\begin{aligned}
\langle\forall C: C \triangleleft D: & : \text { hterm }\left(C, v_{c}\right) \wedge\left(\text { leavesval }(C)=\vec{M}_{c}\right) \wedge \\
& \text { hfinished }\left(C, v_{c}\right)
\end{aligned} \\
\begin{aligned}
\left.\vee \neg\left\langle\forall C: C \triangleleft D:: \text { leavesval }(C)=\vec{M}_{c}\right\rangle\right]
\end{aligned}
\end{gathered}
$$

Weakening the right side:

$\left\langle\forall C: C \triangleleft D::\right.$ hfinished $\left(C, v_{c}\right) \wedge\left(\right.$ leavesval $\left.\left.(C)=\vec{M}_{c}\right)\right\rangle \mapsto$

$\left(\left\langle\forall C: C \triangleleft D:: \operatorname{hterm}\left(C, v_{c}\right)\right\rangle\right.$

$\vee \neg\left\langle\forall C: C \triangleleft D::\right.$ leavesval $\left.\left.(C)=\vec{M}_{c}\right\rangle\right)$

\section{Lemma B.3.}

$$
\begin{gathered}
\left\langle\forall C: C \triangleleft D:: \text { hterm }\left(C, v_{c}\right) \wedge\left(\text { leavesval }(C)=\vec{M}_{c}\right)\right\rangle \mapsto \\
(\langle\exists v:: \text { hfinished }(D, v)\rangle \\
\left.\vee \neg\left\langle\forall C: C \triangleleft D:: \text { leavesval }(C)=\vec{M}_{c}\right\rangle\right) .
\end{gathered}
$$

Proof.

From SP3, using Simple Conjunction:

$$
\begin{gathered}
\left\langle\forall C: C \triangleleft D:: \text { hinished }\left(C, v_{c}\right) \wedge\left(\text { leavesval }(C)=\vec{M}_{c}\right)\right\rangle \text { unless } \\
\neg\left\langle\forall C: C \triangleleft D:: \text { leavesval }(C)=\vec{M}_{c}\right\rangle
\end{gathered}
$$

The PSP theorem on SP5 and the above:

$$
\begin{gathered}
\left\langle\forall C: C \triangleleft D:: \text { hterm }\left(C, v_{c}\right) \wedge \text { hfinished }\left(C, v_{c}\right) \wedge\left(\text { leavesval }(C)=\vec{M}_{c}\right)\right\rangle \mapsto \\
{\left[\left(\langle\exists v:: \text { hinished }(D, v)\rangle \vee \neg\left\langle\forall C: C \triangleleft D:: \text { hinished }\left(C, v_{c}\right)\right\rangle\right) \wedge\right.} \\
\left.\left\langle\forall C: C \triangleleft D:: \text { hfinished }\left(C, v_{c}\right) \wedge\left(\text { leavesval }(C)=\vec{M}_{c}\right)\right\rangle\right) \\
\left.\vee \neg\left\langle\forall C: C \triangleleft D:: \text { leavesval }(C)=\vec{M}_{c}\right\rangle\right]
\end{gathered}
$$

Rewriting the left- and right side:

$$
\begin{aligned}
& \left\langle\forall C: C \triangleleft D:: \text { hterm }\left(C, v_{c}\right) \wedge\left(\text { leavesval }(C)=\vec{M}_{c}\right)\right\rangle \mapsto \\
& {[(\langle\exists v:: \text { hinished }(D, v)\rangle \wedge} \\
& \left.\quad\left\langle\forall C: C \triangleleft D:: \text { hfinished }\left(C, v_{c}\right) \wedge\left(\text { leavesval }(C)=\vec{M}_{c}\right)\right\rangle\right) \\
& \left.\quad \vee \neg\left\langle\forall C: C \triangleleft D:: \text { leavesval }(C)=\vec{M}_{c}\right\rangle\right]
\end{aligned}
$$

Weakening the right side:

$$
\begin{aligned}
& \left\langle\forall C: C \triangleleft D:: \text { hterm }\left(C, v_{c}\right) \wedge\left(\text { leavesval }(C)=\vec{M}_{c}\right)\right\rangle \mapsto \\
& \quad\left(\langle\exists v:: \text { hfinished }(D, v)\rangle \vee \neg\left\langle\forall C: C \triangleleft D:: \text { leavesval }(C)=\vec{M}_{c}\right\rangle\right)
\end{aligned}
$$

Lemma B.4. For all domains $D$ :

$$
\text { (leavesval }(D)=\vec{M}) \mapsto(\langle\exists v \quad: \text { hifinished }(D, v)\rangle \vee \neg(\text { leavesval }(D)=\vec{M})) \text {. }
$$

Proof. We will give a proof by induction on the structure of the division-tree. 
Base: If $D$ is a leaf, this case trivially follows from SP5.

Step: Suppose $D$ is an internal node.

From Induction Hypothesis:

$\left\langle\forall C: C \triangleleft D::\left(\right.\right.$ leavesval $\left.(C)=\vec{M}_{c}\right) \mapsto$

$$
\left(\left\langle\exists v_{c}:: \text { hfinished }\left(C, v_{c}\right)\right\rangle \vee \neg\left(\text { leavesval }(C)=\vec{M}_{c}\right)\right)
$$

Rewriting the right side:

$$
\begin{aligned}
\langle\forall C: C \triangleleft D:: & \text { leavesval } \left.(C)=\vec{M}_{c}\right) \mapsto \\
& \left(\left\langle\exists v_{c}:: \text { hfinished }\left(C, v_{c}\right)\right\rangle \wedge\left(\text { leavesval }(C)=\vec{M}_{c}\right)\right) \\
& \vee \neg\left(\text { leavesval }(C)=\vec{M}_{c}\right)
\end{aligned}
$$

From SP3:

$\left\langle\forall C: C \triangleleft D::\left(\right.\right.$ hfinished $\left(C, v_{c}\right) \wedge\left(\right.$ leavesval $\left.\left.(C)=\vec{M}_{c}\right)\right)$ unless

$$
\neg\left(\text { leavesval }(C)=\vec{M}_{c}\right)
$$

Using the Generalization of the Completion theorem on the above two relations:

$$
\begin{aligned}
&\left\langle\forall C: C \triangleleft D:: \text { leavesval }(C)=\vec{M}_{c}\right\rangle \mapsto \\
& {\left[\left\langle\forall C: C \triangleleft D::\left\langle\exists v_{c}:: \text { hinished }\left(C, v_{c}\right)\right\rangle \wedge\left(\text { leavesval }(C)=\vec{M}_{c}\right)\right\rangle\right.} \\
&\left.\vee \neg\left\langle\forall C: C \triangleleft D:: \text { leavesval }(C)=\vec{M}_{c}\right\rangle\right]
\end{aligned}
$$

The Cancellation theorem on the result of Lemma B.2 and the above:

$$
\begin{aligned}
\left\langle\forall C: C \triangleleft D:: \text { leavesval }(C)=\vec{M}_{c}\right\rangle \mapsto \\
\quad\left(\left\langle\forall C: C \triangleleft D::\left\langle\exists v_{c}:: \text { hterm }\left(C, v_{c}\right)\right\rangle\right\rangle\right. \\
\left.\quad \vee \neg\left\langle\forall C: C \triangleleft D:: \text { leavesval }(C)=\vec{M}_{c}\right\rangle\right)
\end{aligned}
$$

Rewriting the right side:

$$
\begin{aligned}
& \left\langle\forall C: C \triangleleft D:: \text { leavesval }(C)=\vec{M}_{c}\right\rangle \mapsto \\
& \quad\left(\left\langle\forall C: C \triangleleft D::\left\langle\exists v_{c}:: \text { hterm }\left(C, v_{c}\right)\right\rangle \wedge\left(\text { leavesval }(C)=\vec{M}_{c}\right)\right\rangle\right. \\
& \left.\quad \vee \neg\left\langle\forall C: C \triangleleft D:: \text { leavesval }(C)=\vec{M}_{c}\right\rangle\right)
\end{aligned}
$$

Using the Cancellation theorem on the result of Lemma B.3 and the above:

$$
\begin{aligned}
& \left\langle\forall C: C \triangleleft D:: \text { leavesval }(C)=\vec{M}_{c}\right\rangle \mapsto \\
& \quad\left(\langle\exists v:: \text { hinished }(D, v)\rangle \vee \neg\left\langle\forall C: C \triangleleft D:: \text { leavesval }(C)=\vec{M}_{c}\right\rangle\right)
\end{aligned}
$$

Rewriting the left- and right side concludes the induction step, thereby proving the lemma.

Theorem B.1. SP2 is met by SP3, SP4, SP5 and SP6.

Proof. Let $R$ be the root of the division tree, then from Lemma B.4 and Corollary B.1:

$$
(\text { leavesval }(R)=\vec{M}) \mapsto(\langle\exists v \quad:: \text { hterm }(R, v)\rangle \vee \neg(\text { leavesval }(R)=\vec{M}))
$$

Because $($ leavesval $(R)=\operatorname{nodesval}(G))$ and $(\operatorname{hterm}(R, v)=\operatorname{term}(G, v))$, SP2 follows. 


\section{Formal Proof of Refined Solution Strategy for Division Trees}

It is proven that any strategy satisfying conditions SP3, SP4, SP7, SP8 and SP9 (Section 5.2.4) also meets the conditions SP3, SP4, SP5 and SP6 (Section 4.1.2). In the proof a number of known results are used, which are given in Appendix $\mathrm{A}$.

Theorem C.1. SP5 is met by SP3, SP4, SP7, SP8 and SP9.

Proof. Two cases are considered:

1. $\operatorname{leaves}(D)=\{D\}$ :

This case directly follows from SP7.

2. leaves $(D) \neq\{D\}$ :

Simple Conjunction of SP4:

$\left\langle\forall C: C \triangleleft D::\right.$ hterm $\left.\left(C, v_{c}\right)\right\rangle$ unless $\left\langle\exists C: C \triangleleft D::\right.$ - $:$ hfinished $\left.\left(C, v_{c}\right)\right\rangle$

PSP on the above and SP8:

$$
\begin{aligned}
\langle\forall C: & \left.C \triangleleft D:: \text { hterm }\left(C, v_{c}\right)\right\rangle \mapsto \\
& {\left[\left(\left\langle\forall C: C \triangleleft D:: \text { hterm }\left(C, v_{c}\right)\right\rangle \wedge\right.\right.} \\
& \left.\left(m^{D}(D)=\left\langle\max C: C \triangleleft D:: m^{D}(C)\right\rangle\right)\right) \\
& \left.\vee\left\langle\exists C: C \triangleleft D:: \text { - } \backslash \text { hfinished }\left(C, v_{c}\right)\right\rangle\right]
\end{aligned}
$$

Rewriting the right side:

$$
\begin{aligned}
& \left\langle\forall C: C \triangleleft D:: \text { hterm }\left(C, v_{c}\right)\right\rangle \mapsto \\
& \quad\left(\langle\exists v: \text { hfinished }(D, v)\rangle \vee\left\langle\exists C: C \triangleleft D:: \neg \text { hfinished }\left(C, v_{c}\right)\right\rangle\right)
\end{aligned}
$$

Theorem C.2. SP6 is met by SP3, SP4, SP7, SP8 and SP9.

Proof.

Antireflexivity of unless:

hfinished $(D, v)$ unless $\neg$ hinished $(D, v)$

PSP on the above and SP7:

hfinished $(D, v) \mapsto$

$\left[\left(\right.\right.$ hinished $(D, v) \wedge$ fatherknows $\left.\left(D, m^{D}(D)\right)\right) \vee \neg$ finished $\left.(D, v)\right]$

Rewriting the right side:

$h$ finished $(D, v) \mapsto[\operatorname{hterm}(D, v) \vee \neg$ finished $(D, v)]$

\section{Formal Proof of Refined Solution Strategy for Hierarchically Divided Graphs}

It is proven that any strategy satisfying conditions SP14 through SP22 (Section 6.2.4) also meet the conditions SP10, SP11, SP12 and SP13 (Section 5.2.2). In the proof a number of known results are used, which are given in appendix $A$.

Theorem D.1. SP10 is met by SP14, SP15, SP16, SP17, SP18, SP19, SP20, SP21 and $\mathrm{SP} 22$.

Proof. To prove the theorem, we use induction on the structure of the hierarchical division.

Base: Dnodes $(D)=\{D\}$. From SP14, this case directly follows.

Step: Suppose Dnodes $(D) \neq\{D\}$. 
From Induction Hypothesis:

$\left\langle\forall C: C \triangleleft D::\left(\right.\right.$ Dhfinished $\left(C, v_{c}\right) \wedge\left(\right.$ Dnodesval $\left.\left.(C)=\vec{M}_{c}\right)\right)$ unless $\neg\left(\right.$ Dnodesval $\left.(C)=\vec{M}_{c}\right)$

Simple Conjunction on the above, and rewriting the left and right side:

$\left(\left\langle\forall C: C \triangleleft D::\right.\right.$ Dhfinished $\left.\left(C, v_{c}\right)\right\rangle \wedge($ Dnodesval $\left.(D)=\vec{M})\right)$ unless $\neg($ Dnodesval $(D)=\vec{M})$

From SP15, using Simple Conjunction over all $n \in \operatorname{Dnodes}(D)$, and rewriting the left term:

$\left(\left\langle\forall C: C \triangleleft D::\right.\right.$ Dhterm $\left.\left(C, v_{c}\right)\right\rangle \wedge$

$\langle\forall n: n \in \operatorname{Dnodes}(D)::\langle\exists v \quad::$ Nfinished $(n, D, v)\rangle\rangle)$ unless $\left\langle\exists C: C \triangleleft D:: \neg D\right.$ Dhinished $\left.\left(C, v_{c}\right)\right\rangle$

Rewriting the left term:

$\left(\left\langle\forall C: C \triangleleft D::\right.\right.$ Dhterm $\left.\left.\left(C, v_{c}\right)\right\rangle \wedge \operatorname{Dhfinished}(D, v)\right)$ unless

$\left\langle\exists C: C \triangleleft D:: \neg\right.$ Dhfinished $\left.\left(C, v_{c}\right)\right\rangle$

Let $(\operatorname{Dnodesval}(D)=\vec{M})$, then rewrite the left term:

$\left(\right.$ Dhfinished $(D, v) \wedge\left\langle\forall C: C \triangleleft D::\right.$ Dhterm $\left.\left(C, v_{c}\right)\right\rangle \wedge$

(Dnodesval $(D)=\vec{M})$ ) unless

$\left\langle\exists C: C \triangleleft D:: \neg\right.$ Dhfinished $\left.\left(C, v_{c}\right)\right\rangle$

Conjunction on the above and prf1, and rewriting the left term:

$\left(\right.$ Dhfinished $(D, v) \wedge\left\langle\forall C: C \triangleleft D::\right.$ Dhterm $\left.\left(C, v_{c}\right)\right\rangle \wedge$

(Dnodesval $(D)=\vec{M}))$ unless

$\left[\left(\operatorname{Dhfinished}(D, v) \wedge\left\langle\forall C: C \triangleleft D::\right.\right.\right.$ Dhterm $\left.\left(C, v_{c}\right)\right\rangle \wedge$

$($ Dnodesval $(D)=\vec{M}) \wedge \neg($ Dnodesval $(D)=\vec{M})) \vee$

$\left(\left\langle\forall C: C \triangleleft D::\right.\right.$ Dhfinished $\left.\left(C, v_{c}\right)\right\rangle \wedge($ Dnodesval $(D)=\vec{M}) \wedge$

$\left\langle\exists C: C \triangleleft D:: \neg D\right.$ hifinished $\left.\left.\left(C, v_{c}\right)\right\rangle\right) \vee$

$\left(\left\langle\exists C: C \triangleleft D:: \neg\right.\right.$ Dhfinished $\left.\left(C, v_{c}\right)\right\rangle \wedge \neg($ Dnodesval $\left.\left.(D)=\vec{M})\right)\right]$

Rewriting the left and right side:

$(\operatorname{Dhfinished}(D, v) \wedge(\operatorname{Dnodesval}(D)=\vec{M}))$ unless $\neg($ Dnodesval $(D)=\vec{M})$

This concludes the induction step, thereby proving the theorem.

Theorem D.2. SP11 is met by SP14, SP15, SP16, SP17, SP18, SP19, SP20, SP21 and SP22.

Proof.

Simple Conjunction of SP16 and SP17:

$\left(\right.$ Dhfinished $(D, v) \wedge\left\langle\wedge n: n \in\right.$ Dnodes $\left.(D):: m_{n}^{f(D)}(D)=m_{n}^{D}(D)\right\rangle \wedge$

equal $(D, S))$ unless $\neg D$ hinished $(D, v)$

Substitution on the above, using Invariant SP22:

$\operatorname{Dhterm}(D, v)$ unless $\neg$ Dhfinished $(D, v)$

Theorem D.3. SP12 is met by SP14, SP15, SP16, SP17, SP18, SP19, SP20, SP21 and SP22.

Proof. Two cases are distinguished: 
1. $D \in \operatorname{Dnodes}(D)$ :

From SP18, this case directly follows.

2. $\neg(D \in \operatorname{Dnodes}(D))$ :

From Theorem D.2

$\operatorname{Dhterm}\left(C, v_{c}\right)$ unless $\neg$ Dhfinished $\left(C, v_{c}\right)$

From above, using Simple Conjunction:

$\left\langle\forall C: C \triangleleft D::\right.$ Dhterm $\left.\left(C, v_{c}\right)\right\rangle$ unless

From SP18:

$$
\left\langle\exists C: C \triangleleft D:: \neg D \text { Dhinished }\left(C, v_{c}\right)\right\rangle
$$

$$
(n \in \operatorname{Dnodes}(D) \wedge n \neq D) \mapsto\langle\exists v:: \text { Nfinished }(n, D, v)\rangle)
$$

The PSP theorem on the above two:

$\left(n \in \operatorname{Dnodes}(D) \wedge n \neq D \wedge\left\langle\forall C: C \triangleleft D::\right.\right.$ Dhterm $\left.\left.\left(C, v_{c}\right)\right\rangle\right) \mapsto$ $\left[\left(\left\langle\forall C: C \triangleleft D::\right.\right.\right.$ Dhterm $\left.\left(C, v_{c}\right)\right\rangle \wedge\langle\exists v::$ Nfinished $\left.(n, D, v)\rangle\right)$

$\left.\vee\left\langle\exists C: C \triangleleft D:: \neg D h f i n i s h e d\left(C, v_{c}\right)\right\rangle\right]$

Conjunction over the $n$ 's on the above:

$\langle\forall n: n \in$ Dnodes $(D) \wedge n \neq D::$

$\left\langle\forall C: C \triangleleft D::\right.$ Dhterm $\left.\left(C, v_{c}\right)\right\rangle \mapsto$ $\left[\left(\left\langle\forall C: C \triangleleft D::\right.\right.\right.$ Dhterm $\left.\left(C, v_{c}\right)\right\rangle \wedge\langle\exists v::$ Nfinished $\left.(n, D, v)\rangle\right)$ $\vee\left\langle\exists C: C \triangleleft D:: \neg\right.$ Dhfinished $\left.\left.\left(C, v_{c}\right)\right\rangle\right]$

)

From SP15, using Simple Conjunction:

$\langle\forall n: n \in$ Dnodes $(D) \wedge n \neq D::$

$\left(\left\langle\forall C: C \triangleleft D::\right.\right.$ Dhterm $\left.\left(C, v_{c}\right)\right\rangle \wedge\langle\exists v::$ Nfinished $\left.(n, D, v)\rangle\right)$ unless

$\left\langle\exists C: C \triangleleft D:: \neg \operatorname{Dhfinished}\left(C, v_{c}\right)\right\rangle$

The Completion theorem on the above two:

$\left\langle\forall C: C \triangleleft D:: \operatorname{Dhterm}\left(C, v_{c}\right)\right\rangle \mapsto$

$$
\begin{aligned}
& {\left[\left(\left\langle\forall C: C \triangleleft D:: D \text { Dhterm }\left(C, v_{c}\right)\right\rangle \wedge\right.\right.} \\
& \langle\wedge n: n \in D \text { Dnodes }(D) \wedge n \neq D::\langle\exists v:: \text { Nfinished }(n, D, v)\rangle\rangle) \\
& \left.\vee\left\langle\exists C: C \triangleleft D:: \neg D \text { Dhinished }\left(C, v_{c}\right)\right\rangle\right]
\end{aligned}
$$

Rewriting the right side:

$\left\langle\forall C: \dot{C} \triangleleft D::\right.$ Dhterm $\left.\left(C, v_{c}\right)\right\rangle \mapsto$

$\left(\langle\exists v::\right.$ Dhfinished $(D, v)\rangle \vee\left\langle\exists C: C \triangleleft D:: \neg\right.$ Dhfinished $\left.\left(C, v_{c}\right)\right\rangle$

Lemma D.1. For all domains $D$ :

Dhfinished $(D, v) \mapsto$

$\left[\left(D h f i n i s h e d(D, v) \wedge\left\langle\wedge n: n \in\right.\right.\right.$ Dnodes $\left.\left.(D):: m_{n}^{f(D)}(D)=m_{n}^{D}(D)\right\rangle\right)$

$\vee \neg \operatorname{Dhfinished}(D, v)]$.

Proof.

From SP19:

$(n \in \operatorname{Dnodes}(D)) \mapsto\left(m_{n}^{f(D)}(D)=m_{n}^{D}(D)\right)$

Antireflexivity of unless:

Dhfinished $(D, v)$ unless $\neg$ Dhfinished $(D, v)$

The PSP theorem on the above two:

$[(n \in \operatorname{Dnodes}(D)) \wedge \operatorname{Dhfinished}(D, v)] \mapsto$

$\left[\left(\operatorname{Dhfinished}(D, v) \wedge\left(m_{n}^{f(D)}(D)=m_{n}^{D}(D)\right)\right) \vee \neg\right.$ Dhfinished $\left.(D, v)\right]$

Conjunction over the $n$ 's on the above: 
$\langle\wedge n: n \in \operatorname{Dnodes}(D)::$

$[(n \in \operatorname{Dnodes}(D)) \wedge$ Dhfinished $(D, v)] \mapsto$

)

$\left[\left(\right.\right.$ Dhfinished $\left.(D, v) \wedge\left(m_{n}^{f(D)}(D)=m_{n}^{D}(D)\right)\right) \vee \neg$ Dhfinished $\left.(D, v)\right]$

From SP16, using Simple Conjunction:

$\langle\wedge n: n \in$ Dnodes $(D)::$

$\left(\right.$ Dhfinished $\left.(D, v) \wedge\left(m_{n}^{f(D)}(D)=m_{n}^{D}(D)\right)\right)$ unless $\neg$ Dhfinished $(D, v)$

The Completion theorem on the above two relations, and rewriting the left and right side:

Dhfinished $(D, v) \mapsto$

$\left[\left(\operatorname{Dhfinished}(D, v) \wedge\left\langle\wedge n: n \in \operatorname{Dnodes}(D):: m_{n}^{f(D)}(D)=m_{n}^{D}(D)\right\rangle\right)\right.$

$\vee \neg D$ Dhinished $(D, v)]$

Lemma D.2. For all domains $D$ :

$$
\begin{gathered}
\left(\left\{n, n^{\prime}\right\} \in E \wedge\left(n, n^{\prime} \in \text { Dnodes }(f(D))\right) \wedge\left(S_{n}(D)=S\right)\right) \mapsto \\
{\left[\left(S_{n}(D)=S_{n^{\prime}}(D)=S\right) \vee\left(\max \left\{S_{n}(D), S_{n^{\prime}}(D)\right\}>S\right)\right] .}
\end{gathered}
$$

Proof.

From SP21:

$\left(\left\{n, n^{\prime}\right\} \in E \wedge\left(n, n^{\prime} \in \operatorname{Dnodes}(f(D))\right)\right) \mapsto\left(S_{n^{\prime}}(D) \geq S_{n}(D)\right)$

The PSP theorem on SP20 and the above:

$\left(\left\{n, n^{\prime}\right\} \in E \wedge\left(n, n^{\prime} \in \operatorname{Dnodes}(f(D))\right) \wedge\left(S_{n}(D)=S\right)\right) \mapsto$

$$
\left[\left(\left(S_{n^{\prime}}(D) \geq S_{n}(D)\right) \wedge\left(S_{n}(D)=S\right)\right) \vee\left(S_{n}(D)>S\right)\right]
$$

Rewriting the right side:

$\left(\left\{n, n^{\prime}\right\} \in E \wedge\left(n, n^{\prime} \in \operatorname{Dnodes}(f(D))\right) \wedge\left(S_{n}(D)=S\right)\right) \mapsto$

$$
\left[\left(S_{n}(D)=S_{n^{\prime}}(D)=S\right) \vee\left(\max \left\{S_{n}(D), S_{n^{\prime}}(D)\right\}>S\right)\right]
$$

Lemma D.3. For all domains $D$ :

$$
\operatorname{maxs}(D, S) \mapsto\left(\operatorname{equal}(D, S) \vee\left(\operatorname{maxs}\left(D, S^{\prime}\right) \wedge\left(S^{\prime}>S\right)\right)\right)
$$

Proof. Let

$$
\begin{aligned}
& \operatorname{conin}\left(f(D), n_{1}, \ldots, n_{x}\right)= \\
& \quad\left(\left\langle\forall i: 1 \leq i \leq x:: n_{i} \in \operatorname{Dnodes}(f(D))\right\rangle \wedge\right. \\
& \quad\left[(x=1) \vee\left\langle\forall i: 1 \leq i \leq x::\left\langle\exists j: 1 \leq j \leq x \wedge i \neq j::\left\{n_{i}, n_{j}\right\} \in E\right\rangle\right]\right) .
\end{aligned}
$$

In other words, conin $\left(f(D), n_{1}, \ldots, n_{x}\right)$ holds if and only if every node in the set $\left\{n_{1}, \ldots, n_{x}\right\}$ is contained in $f(D)$ and has a neighbour in the set.

We use induction on the number of nodes to prove:

$\left[\operatorname{conin}\left(f(D), n_{1}, \ldots, n_{x}\right) \wedge\left(\max \left\{S_{n_{1}}(D), \ldots, S_{n_{x}}(D)\right\}=S\right)\right] \mapsto$

$$
\left[\left(S_{n_{1}}(D)=\ldots=S_{n_{x}}(D)=S\right) \vee\left(\max \left\{S_{n_{1}}(D), \ldots, S_{n_{x}}(D)\right\}>S\right)\right]
$$

Base: $x=1$. Follows directly from the Implication theorem on $\left(\max \left\{S_{n_{1}}(D)\right\}=S\right) \Rightarrow\left(S_{n_{1}}(D)=S\right)$.

Step: Suppose conin $\left(f(D), n, n_{1}, \ldots, n_{y}\right),\left\{n_{i}, n\right\} \in E$ and

$\left[\operatorname{conin}\left(f(D), n_{1}, \ldots, n_{y}\right) \wedge\left(\max \left\{S_{n_{1}}(D), \ldots, S_{n_{y}}(D)\right\}=S\right)\right] \mapsto$

$$
\left[\left(S_{n_{1}}(D)=\ldots=S_{n_{y}}(D)=S\right) \vee\left(\max \left\{S_{n_{1}}(D), \ldots, S_{n_{y}}(D)\right\}>S\right)\right] \text {. }
$$


Then the following holds:

From Lemma D.2 and $\left\{n, n_{i}\right\} \in E$ :

$\left(\operatorname{conin}\left(f(D), n, n_{1}, \ldots, n_{y}\right) \wedge\left(S_{n}(D)=S\right)\right) \mapsto$

$$
\left[\left(S_{n}(D)=S_{n_{i}}(D)=S\right) \vee\left(\max \left\{S_{n}(D), S_{n_{i}}(D)\right\}>S\right)\right]
$$

Using the Implication theorem:

$$
\begin{gathered}
{\left[\left(S_{n}(D)=S_{n_{i}}(D)=S\right) \vee\left(\max \left\{S_{n}(D), S_{n_{i}}(D)\right\}>S\right)\right] \mapsto} \\
{\left[\left(\left(\max \left\{S_{n_{1}}(D), \ldots, S_{n_{y}}(D)\right\}=S\right) \wedge\left(S_{n}(D)=S\right)\right)\right.} \\
\left.\vee\left(\max \left\{S_{n_{1}}(D), \ldots, S_{n_{y}}(D), S_{n}(D)\right\}>S\right)\right]
\end{gathered}
$$

Transitivity on the above two:

$$
\begin{gathered}
\left(\operatorname{conin}\left(f(D), n, n_{1}, \ldots, n_{y}\right) \wedge\left(S_{n}(D)=S\right)\right) \mapsto \\
{\left[\left(\left(\max \left\{S_{n_{1}}(D), \ldots, S_{n_{y}}(D)\right\}=S\right) \wedge\left(S_{n}(D)=S\right)\right)\right.} \\
\left.\quad \vee\left(\max \left\{S_{n_{1}}(D), \ldots, S_{n_{y}}(D), S_{n}(D)\right\}>S\right)\right]
\end{gathered}
$$

From the Induction Hypothesis:

$$
\left(\operatorname{conin}\left(f(D), n, n_{1}, \ldots, n_{y}\right) \wedge\left(\max \left\{S_{n_{1}}(D), \ldots, S_{n_{y}}(D)\right\}=S\right)\right) \mapsto
$$

$$
\begin{aligned}
& {\left[\left(S_{n_{1}}(D)=\ldots=S_{n_{y}}(D)=S\right)\right.} \\
& \left.\quad \vee\left(\max \left\{S_{n_{1}}(D), \ldots, S_{n_{y}}(D)\right\}>S\right)\right]
\end{aligned}
$$

The PSP theorem on the above and SP20:

$$
\left[\operatorname{conin}\left(f(D), n, n_{1}, \ldots, n_{y}\right) \wedge\left(\max \left\{S_{n_{1}}(D), \ldots, S_{n_{y}}(D)\right\}=S\right) \wedge\right.
$$

$$
\begin{aligned}
& \left.\left(S_{n}(D)=S\right)\right] \mapsto \\
& {\left[\left(\left[\left(S_{n_{1}}(D)=\ldots=S_{n_{y}}(D)=S\right) \vee\left(\max \left\{S_{n_{1}}(D), \ldots, S_{n_{y}}(D)\right\}>S\right)\right] \wedge\right.\right.} \\
& \left.\quad\left(S_{n}(D)=S\right)\right) \\
& \left.\vee\left(S_{n}(D)>S\right)\right]
\end{aligned}
$$

Rewrite the right side:

$\left[\operatorname{conin}\left(f(D), n, n_{1}, \ldots, n_{y}\right) \wedge\left(\max \left\{S_{n_{1}}(D), \ldots, S_{n_{p}}(D)\right\}=S\right) \wedge\right.$

$$
\left.\left(S_{n}(D)=S\right)\right] \mapsto
$$

$$
\begin{aligned}
& {\left[\left(S_{n_{1}}(D)=\ldots=S_{n_{y}}(D)=S_{n}(D)=S\right)\right.} \\
& \left.\vee\left(\max \left\{S_{n_{1}}(D), \ldots, S_{n_{y}}(D), S_{n}(D)\right\}>S\right)\right]
\end{aligned}
$$

Rewrite the right side of prf1:

$$
\left(\operatorname{conin}\left(f(D), n, n_{1}, \ldots, n_{y}\right) \wedge\left(S_{n}(D)=S\right)\right) \mapsto
$$

$$
\begin{aligned}
& {\left[\left(\operatorname{conin}\left(f(D), n, n_{1}, \ldots, n_{y}\right) \wedge\left(\max \left\{S_{n_{1}}(D), \ldots, S_{n_{y}}(D)\right\}=S\right) \wedge\right.\right.} \\
& \left.\left(S_{n}(D)=S\right)\right) \\
& \left.\vee\left(\max \left\{S_{n_{1}}(D), \ldots, S_{n_{y}}(D), S_{n}(D)\right\}>S\right)\right]
\end{aligned}
$$

The Cancellation theorem on the above two:

$\left(\operatorname{conin}\left(f(D), n, n_{1}, \ldots, n_{y}\right) \wedge\left(S_{n}(D)=S\right)\right) \mapsto$

$$
\begin{aligned}
& {\left[\left(S_{n_{1}}(D)=\ldots=S_{n_{y}}(D)=S_{n}(D)=S\right)\right.} \\
& \left.\vee\left(\max \left\{S_{n_{1}}(D), \ldots, S_{n_{y}}(D), S_{n}(D)\right\}>S\right)\right]
\end{aligned}
$$

From SP20, using Simple Conjunction:

$$
\left(S_{n_{1}}(D)=\ldots=S_{n_{y}}(D)=S\right) \text { unless }\left[\left(S_{n_{1}}(D)>S\right) \vee \ldots \vee\left(S_{n_{y}}(D)>S\right)\right]
$$

Rewriting the right side:

$$
\left(S_{n_{1}}(D)=\ldots=S_{n_{y}}(D)=S\right) \text { unless }\left[\max \left\{S_{n_{1}}(D), \ldots, S_{n_{y}}(D)\right\}>S\right]
$$

From Lemma D.2 and $\left\{n, n_{i}\right\} \in E$ :

$$
\begin{aligned}
& \left(\operatorname{conin}\left(f(D), n, n_{1}, \ldots, n_{y}\right) \wedge\left(S_{n_{i}}(D)=S\right)\right) \mapsto \\
& {\left[\left(S_{n_{i}}(D)=S_{n}(D)=S\right) \vee\left(\max \left\{S_{n}(D), S_{n_{i}}(D)\right\}>S\right)\right]}
\end{aligned}
$$

The PSP theorem on the above two:

$$
\begin{aligned}
& \left(\operatorname{conin}\left(f(D), n, n_{1}, \ldots, n_{y}\right) \wedge\left(S_{n_{1}}(D)=\ldots=S_{n_{y}}(D)=S\right)\right) \mapsto \\
& \quad\left[\left(\left[\left(S_{n_{n}}(D)=S_{n}(D)=S\right) \vee\left(\max \left\{S_{n}(D), S_{n_{i}}(D)\right\}>S\right)\right] \wedge\right.\right. \\
& \left.\quad\left(S_{n_{1}}(D)=\ldots=S_{n_{y}}(D)=S\right)\right) \\
& \left.\quad \vee\left(\max \left\{S_{n_{1}}(D), \ldots, S_{n_{y}}(D)\right\}>S\right)\right]
\end{aligned}
$$

Rewriting the right side:

$$
\left(\operatorname{conin}\left(f(D), n, n_{1}, \ldots, n_{y}\right) \wedge\left(S_{n_{1}}(D)=\ldots=S_{n_{y}}(D)=S\right)\right) \mapsto
$$




$$
\begin{aligned}
& {\left[\left(S_{n_{1}}(D)=\ldots=S_{n_{y}}(D)=S_{n}(D)=S\right)\right.} \\
& \left.\vee\left(\max \left\{S_{n_{1}}(D), \ldots, S_{n_{y}}(D), S_{n}(D)\right\}>S\right)\right]
\end{aligned}
$$

From the Induction Hypothesis and rewriting the right side:

$$
\begin{aligned}
&(\operatorname{conin}\left.\left(f(D), n, n_{1}, \ldots, n_{y}\right) \wedge\left(\max \left\{S_{n_{1}}(D), \ldots, S_{n_{y}}(D)\right\}=S\right)\right) \mapsto \\
& {\left[\left(\operatorname{conin}\left(f(D), n_{1}, \ldots, n_{1}, \ldots, n_{y}\right) \wedge\left(S_{n_{1}}(D)=\ldots=S_{n_{y}}(D)=S\right)\right)\right.} \\
&\left.\quad \vee\left(\max \left\{S_{n_{1}}(D), \ldots, S_{n_{y}}(D)\right\}>S\right)\right]
\end{aligned}
$$

The Cancellation theorem on the above two and rewriting the right side:

$\left(\operatorname{conin}\left(f(D), n, n_{1}, \ldots, n_{y}\right) \wedge\left(\max \left\{S_{n_{1}}(D), \ldots, S_{n_{y}}(D)\right\}=S\right) \mapsto\right.$

$$
\begin{aligned}
& {\left[\left(S_{n_{1}}(D)=\ldots=S_{n_{y}}(D)=S_{n}(D)=S\right)\right.} \\
& \left.\vee\left(\max \left\{S_{n_{1}}(D), \ldots, S_{n_{y}}(D), S_{n}(D)\right\}>S\right)\right]
\end{aligned}
$$

Using the Implication theorem:

$\left(\operatorname{conin}\left(f(D), n, n_{1}, \ldots, n_{y}\right) \wedge\left(\max \left\{S_{n_{1}}(D), \ldots, S_{n_{y}}(D), S_{n}(D)\right\}=S\right)\right) \mapsto$ $\left[\left(\operatorname{conin}\left(f(D), n, n_{1}, \ldots, n_{y}\right) \wedge\left(\max \left\{S_{n_{1}}(D), \ldots, S_{n_{y}}(D)\right\}=S\right)\right)\right.$ $\left.\vee\left(\operatorname{conin}\left(f(D), n, n_{1}, \ldots, n_{y}\right) \wedge\left(S_{n}(D)=S\right)\right)\right]$

The Cancellation theorem on the above, and prf2 and prf3:

$\left(\operatorname{conin}\left(f(D), n, n_{1}, \ldots, n_{y}\right) \wedge\left(\max \left\{S_{n_{1}}(D), \ldots, S_{n_{y}}(D), S_{n}(D)\right)=S\right)\right\} \mapsto$ $\left[\left(S_{n_{1}}(D)=\ldots=S_{n_{y}}(D)=S_{n}(D)=S\right)\right.$

$\left.\vee\left(\max \left\{S_{n_{1}}(D), \ldots, S_{n_{y}}(D), S_{n}(D)\right\}>S\right)\right]$

This proves the induction step.

Because $f(D)$ is always connected, we can conclude that

$$
\operatorname{maxs}(D, S) \mapsto\left(\operatorname{equal}(D, S) \vee\left(\max s\left(D, S^{\prime}\right) \wedge S^{\prime}>S\right)\right)
$$

Lemma D.4. For all domains $D$ :

Dhfinished $(D, v) \mapsto$

$[(\operatorname{Dhfinished}(D, v) \wedge$ equal $(D, S)) \vee \neg D$ hinished $(D, v)]$.

Proof.

Simple Conjunction of SP17:

$\left\langle\wedge n: n \in\right.$ Dnodes $(f(D)):: \operatorname{maxs}\left(D, S_{n}(D)\right) \wedge$ Dhfinished $\left.(D, v)\right\rangle$ unless $\neg$ Dhfinished $(D, v)$

Rewriting the left side:

$(\operatorname{Dhfinished}(D, v) \wedge \operatorname{maxs}(D, S))$ unless $\neg D$ Dhinished $(D, v)$

The PSP theorem on the result of Lemma D.3 and the above:

$(\operatorname{Dhfinished}(D, v) \wedge \operatorname{maxs}(D, S)) \mapsto$

$\left(\left[\left(\right.\right.\right.$ equal $\left.(D, S) \vee\left(\max s\left(D, S^{\prime}\right) \wedge\left(S^{\prime}>S\right)\right)\right) \wedge$

$(D h f i n i s h e d(D, v) \wedge \operatorname{maxs}(D, S))]$

$\vee \neg \operatorname{Dhfinished}(D, v))$

Rewriting the right side:

$(\operatorname{Dhfinished}(D, v) \wedge \operatorname{maxs}(D, S)) \mapsto$

$[(\operatorname{Dhfinished}(D, v) \wedge$ equal $(D, S)) \vee \neg$ Dhfinished $(D, v)]$

$\langle\exists S: \operatorname{maxs}(D, S)\rangle$, use the Implication theorem:

Dhfinished $(D, v) \mapsto($ Dhfinished $(D, v) \wedge\langle\exists S: \operatorname{maxs}(D, S)\rangle)$

Using Transitivity on the above two:

Dhfinished $(D, v) \mapsto[($ Dhfinished $(D, v) \wedge \operatorname{equal}(D, S)) \vee \neg$ Dhfinished $(D, v)]$ 
Theorem D.4. SP13 is met by SP14, SP15, SP16, SP17, SP18, SP19, SP20, SP21 and SP22.

Proof.

Simple Conjunction of SP16:

$\left(D h f i n i s h e d(D, v) \wedge\left\langle\wedge n: n \in\right.\right.$ Dnodes $\left.\left.(D):: m_{n}^{f(D)}(D)=m_{n}^{D}(D)\right\rangle\right)$ unless $\neg$ Dhfinished $(D, v)$

The PSP theorem on the result of Lemma D.4 and the above:

$\left(\operatorname{Dhfinished}(D, v) \wedge\left\langle\wedge n: n \in \operatorname{Dnodes}(D):: m_{n}^{f(D)}(D)=m_{n}^{D}(D)\right\rangle\right) \mapsto$ $\left[\left(\right.\right.$ Dhfinished $(D, v) \wedge\left\langle\wedge n: n \in \operatorname{Dnodes}(D):: m_{n}^{f(D)}(D)=m_{n}^{D}(D)\right\rangle \wedge$ equal $(D, S))$

$\vee \neg$ Dhfinished $(D, v)]$

Substitution on the above, using Invariant SP22:

$\left(D h f i n i s h e d(D, v) \wedge\left\langle\wedge n: n \in \operatorname{Dnodes}(D):: m_{n}^{f(D)}(D)=m_{n}^{D}(D)\right\rangle\right) \mapsto$ $(\operatorname{Dhterm}(D, v) \vee \neg$ Dhfinished $(D, v))$

Using the Cancellation theorem on the result of Lemma D.1 and the above:

$\operatorname{Dhfinished}(D, v) \mapsto(\operatorname{Dhterm}(D, v) \vee \neg$ Dhfinished $(D, v))$

Received October 1990

Accepted in a revised form in November 1991 by R.C. Backhouse 